\title{
Article \\ Weed Control Efficacy and Crop-Weed Selectivity of a New Bioherbicide WeedLock
}

\author{
Mahmudul Hasan ${ }^{1}\left(\mathbb{D}\right.$, Anis Syahirah Mokhtar ${ }^{2}{ }^{\circledR}$, Adam Mustafa Rosli $^{3}$, Hafizuddin Hamdan $^{3}$, \\ Mst. Motmainna ${ }^{1}$ and Muhammad Saiful Ahmad-Hamdani ${ }^{1, *(1)}$ \\ 1 Department of Crop Science, Faculty of Agriculture, Universiti Putra Malaysia, Serdang 43400, Malaysia; \\ gs53801@student.upm.edu.my (M.H.); gs51794@student.upm.edu.my (M.M.) \\ 2 Department of Plant Protection, Faculty of Agriculture, Universiti Putra Malaysia, Serdang 43400, Malaysia; \\ anissyahirah@upm.edu.my \\ 3 EntoGenex Industries Sdn. Bhd., Kuala Lumpur 50480, Malaysia; adam@entogenex.com (A.M.R.); \\ hafeez@entogenex.com (H.H.) \\ * Correspondence: s_ahmad@upm.edu.my
}

check for updates

Citation: Hasan, M.; Mokhtar, A.S.; Rosli, A.M.; Hamdan, H.; Motmainna, M..; Ahmad-Hamdani, M.S. Weed Control Efficacy and Crop-Weed Selectivity of a New Bioherbicide WeedLock. Agronomy 2021, 11, 1488 https://doi.org/10.3390/ agronomy 11081488

Academic Editor:

Alessandra Carrubba

Received: 14 June 2021

Accepted: 20 July 2021

Published: 27 July 2021

Publisher's Note: MDPI stays neutral with regard to jurisdictional claims in published maps and institutional affiliations.

Copyright: (c) 2021 by the authors. Licensee MDPI, Basel, Switzerland. This article is an open access article distributed under the terms and conditions of the Creative Commons Attribution (CC BY) license (https:/ / creativecommons.org/licenses/by/ $4.0 /)$.
Abstract: Plant-based bioherbicides could be an effective alternative to current chemical herbicides for sustainable agriculture. Therefore, this research evaluated the weed control efficacy and cropweed selectivity of the new plant-derived bioherbicide WeedLock compared to commercial herbicides in glasshouse and field conditions. In the glasshouse, the herbicides applied were WeedLock (672.75, 1345.50, 2691.00 $\left.\mathrm{L} \mathrm{ha}^{-1}\right)$, glyphosate isopropyl-amine, and glufosinate-ammonium $\left(1,2,4 \mathrm{~L} \mathrm{ha}^{-1}\right)$, over the untreated (control) on six weeds and four crops. In the field trial, typical weeds were allowed to grow at a uniform density across plots $(2.5 \times 2.5 \mathrm{~m})$, and WeedLock $\left(1345.50,2691.00 \mathrm{~L} \mathrm{ha}^{-1}\right)$, glyphosate isopropyl-amine, and glufosinate-ammonium $\left(2,4 \mathrm{~L} \mathrm{ha}^{-1}\right)$ were applied along with untreated plot (control). A randomized complete block design was set with four replications for glasshouse and field experiments. WeedLock at $1345.50 \mathrm{~L} \mathrm{ha}^{-1}$ showed efficacy similar to glyphosate isopropyl-amine and glufosinate-ammonium at $2 \mathrm{~L} \mathrm{ha}^{-1}$ for Ageratum conyzoides $\mathrm{L}$. in the glasshouse. Applied herbicides killed all tested crops except Zea mays L. at $1345.50 \mathrm{~L} \mathrm{ha}^{-1}$ (WeedLock). WeedLock showed more than $50 \%$ efficacy at 35 days after spray, while $65 \%$ was produced by glyphosate isopropyl-amine and glufosinate-ammonium compared to the untreated plot (control). WeedLock has excellent potential to control weeds in both glasshouse and field conditions and showed a non-selective character.

Keywords: bioherbicide; sustainable weed management; efficacy; selectivity; glyphosate isopropylamine; glufosinate-ammonium

\section{Introduction}

The need to meet food demand is becoming more urgent, as it is forecasted to increase by $50 \%$ over the next century. Thus, agronomists promote practical tools and approaches to eradicate factors that hamper crop production, including weeds. Weed management in the crop field is a challenging task in agriculture. The aggressive interference of weeds in crop production is well recognized in Malaysia and many countries in the world [1]. The farmers mainly prefer chemical herbicides as they are the most effective and practical way to control weeds due to their high efficacy, specific mode of action, affordable cost, and more rapid return [2]. However, excessive use of synthetic herbicides can increase the number of herbicide-resistant biotypes [3,4], low agricultural produce, environmental pollution, and health hazards $[5,6]$. Conventional commercial herbicide application has thus become inevitable despite its unwelcome side effects. On the other hand, introducing plant-based bioherbicides can play an essential role as a substitute for the chemical dependence of synthetic chemical herbicides to control weeds in sustainable agriculture [7].

Nowadays, plant-based bioherbicides produced from plant extracts, allelochemicals, essential oils, or natural byproducts have shown promising potential against weeds and 
have also received more attention as a weed control strategy because of increasing public awareness about the environment and risk of human health deriving from the chemical herbicides. Several plant extract compounds possess specific inhibiting activity against weed growth but cause no detrimental crop injury [8]. This may be explained by the difference in sensitivity in the target enzymes or specific receptors in weeds that recognize and react with the compounds [9]. Bioherbicides may be effectively utilized in an integrated weed management practice to promote a better crop yield and sustainability [10]. Bioherbicides are generally considered by regulatory authorities to have reduced risk over conventional herbicides $[7,11]$. BioWeed was developed by Barmac, Lidcombe, Australia (derived from Pinus radiata D.Don) and Beloukha was marketed by Grochem, Port Melbourne, Australia (derived from Brassica napus L.) are commercial plant-based non-selective, broad-spectrum, and foliar-applied bioherbicides available in the global market [12,13]. Verdeguer et al. [14] reported that, by 2020, six commercial bioherbicides, i.e., Matratec, GreenMatch, GreenMatchEX, WeedZap, Weed Slayer, and Avenger Weed Killer, derived from essential oils and/or their compounds were registered and available in the USA. Bioherbicides such as BioWeed, Avenger Weed Killer (Avenger Products, LLC, Buford, Georgia) and Weed Slayer (Agresearch International, LLC, McKinney, United States) successfully controlled Ochna serrulata Walp., Digitaria sanguinalis (L.) Scop., and Echinochloa crus-galli (L.) P.Beauv., respectively [12,14].

Inclusively, in Malaysia, a new bioherbicide product WeedLock, which was developed from a plant extract, has been marketed locally by EntoGenex Industries Sdn. Bhd. since 2017 [15]. WeedLock is a non-selective contact herbicide that controls a wide range of weed species. WeedLock is absorbed through the foliage and interferes with the plantss ability to retain moisture and rigidity. Sprayed weeds experienced chlorosis and began to wither within hours with a complete kill within days. The drawback is, WeedLock has been only marketed as a $1 \mathrm{~L}$ ready-to-use formulation, making the product economically less imperative to be used in intensive, large-scale agriculture.

Bioherbicides kill target weed species by inhibiting many physiological activities on weed and destabilization of the cell membrane to cell death $[16,17]$. The phytotoxic potential of essential oils involved chlorosis, the burning of leaves, and plant growth reduction, as well as mitosis inhibition, membrane depolarization, a decrease of chlorophyll content, cellular respiration, and oxidative damage [18]. For instance, Parthenium hysterophorus L. methanol extract has bioherbicidal properties that can hinder the physiological and biochemical mechanism of $A$. conyzoides L., Oryza sativa f. spontanea, and Cyperus iria L. [19]. Bioherbicides might change or hinder the activity of plant growth hormones (auxin, ethylene, and cytokinins) and resulted in growth reduction in plants.

Herbicide efficacy is the resultant of an ideal or expected inhibitory effect of a herbicide on a target weed. It is very crucial to identify the species in a weed community, which can help the farmers to select an effective herbicide for weed control. The efficacy is one of the significant factors to determine appropriate herbicides for weed management $[15,20]$. Bioherbicide's efficacy is the critical restrictive aspect for their implementation. Many elements can influence the efficacy of bioherbicides, such as the bioactive compound/allelochemical content, plant growth stage, formulation type, spray preparation, application method, type of soil, and environmental factors (light, $\mathrm{CO}_{2}$, temperature, humidity) [15]. Selective herbicides should be capable of killing weeds without causing detrimental injury to crops. A selective herbicide is more toxic to one plant group/type than to another [21]. Selectivity characteristics of herbicides used in such a manner when the herbicide is in contact with different plant species can kill or reduce weed growth in a growing crop without damaging the crop or controlling only the unwanted vegetation. A herbicide selectivity for a specific crop is governed by complex interactions between the plant, herbicide, and environment (climate and soil).

Bioherbicides are adequate but need to be regularly incorporated into integrated weed management programs to fully bestow their potential in controlling weeds $[7,11]$. Bioassay performance may help researchers to predict a bioherbicide's potential in glasshouse or 
field conditions [22]. However, the performance of bioherbicide might differ due to the influence of several environmental determinants in glasshouse or field conditions [23]. Although the bioherbicidal potential of many plant crude extracts has been reported, the weed control efficacy and crop-weed selectivity of commercial bioherbicides are scant so far. Therefore, a detailed study of commercial bioherbicide WeedLock under both glasshouse and field conditions warrants due attention to evaluating the efficacy and selectivity. In this context, two experiments were conducted to (a) evaluate and validate weed control efficacy and crop-weed selectivity of new bioherbicide WeedLock in comparison to glyphosate isopropyl-amine and glufosinate-ammonium in glasshouse condition, and (b) determine weed control efficacy of WeedLock in comparison to glyphosate isopropyl-amine and glufosinate-ammonium in the field condition.

\section{Materials and Methods}

\subsection{Glasshouse Experiment}

\subsubsection{Experimental Site}

The evaluation of efficacy and selectivity test was executed from March to June 2019, at the Faculty of Agriculture in Farm 15, Universiti Putra Malaysia $\left(3^{\circ} 02^{\prime} \mathrm{N}\right.$ latitude and $101^{\circ} 42^{\prime} \mathrm{E}$ longitude at $31 \mathrm{~m}$ ), Selangor, Malaysia. The average temperature and relative humidity in the glasshouse were $31.42{ }^{\circ} \mathrm{C}$ and $84 \%$, respectively.

\subsubsection{Test Plants}

Six weed species, namely A. conyzoides, Euphorbia hirta L., Eleusine indica (L.) Gaertn., Axonopus compressus (Sw.) P.Beauv., C. iria, and Fimbristylis miliacea (L.) Vahl, and four crops comprising Oryza sativa L., Z. mays, Abelmoschus esculentus (L.) Moench, and Amaranthus gangeticus L., were used in this research as test plants. The seeds of Z. mays, A. esculentus, and A. gangeticus were purchased from the Green World Genetics Sdn. Bhd., Rawang, Selangor, Malaysia. A local aerobic rice variety O. sativa "MRIA 1" seeds, were collected from MARDI (Malaysian Agricultural Research and Development Institute) in Penang, Malaysia. The weed seeds of A. conyzoides, E. hirta, E. indica, A. compressus, C. iria, and F. miliacea were collected from Farm 15 at the Universiti Putra Malaysia, while A. compressus was grown from the stolon.

\subsubsection{Experimental Treatments and Design}

Seeds from all collected weed species were soaked in $0.2 \%$ potassium nitrate $\left(\mathrm{KNO}_{3}\right)$ for $24 \mathrm{~h}$, then rinsed with distilled water and grown in germination trays. Ten equal-size healthy seedlings were transplanted into plastic trays $(40 \times 30 \times 10 \mathrm{~cm})$ containing potting mix (river sand, peat grow, topsoil at 3:2:1 ratio). Plants were kept well watered and fertilized. When weed species reached the 4-6-leaf stage for broadleaves (A. conyzoides, E. hirta, A. esculentus, A. gangeticus) and 2-3-leaf stage for grasses and sedges (E. indica, A. compressus, C. iria, F. miliacea, and O. sativa, Z. mays), the plants were treated with WeedLock, glyphosate isopropyl-amine, and glufosinate-ammonium. WeedLock is a plantbased ready-to-use bioherbicide developed by EntoGenex Industries Sdn. Bhd. The active ingredient (a.i) of WeedLock is EGX-101 ${ }^{\mathrm{TM}}$, a natural derivative of the Solanum habrochaites S. Knapp and D.M. Spooner (wild tomato) plant. The current formulation of WeedLock contains 10\% EGX-101 and 90\% inert materials. WeedLock was applied at three rates, $672.75,1345.50$ (recommended rate), $2691.00 \mathrm{~L} \mathrm{ha}^{-1}$, and untreated (control). The two commercial tank-mix chemical herbicides, namely Roundup ${ }^{\circledR}$ (glyphosate isopropyl-amine $41 \%$ a.i.; $360 \mathrm{~g}$ glyphosate isopropyl-amine liter $^{-1}$ ) commercialized by Monsanto (Missouri, United States), and Basta ${ }^{\circledR}$ (glufosinate-ammonium 13.5\% a.i.; 150 g glufosinate-ammonium liter $^{-1}$ ) marketed by Bayer Crop Science (Leverkusen, Germany), were applied at three rates, 1, 2 (recommended rate), $4 \mathrm{~L} \mathrm{ha}^{-1}$, and untreated (control). The herbicides were applied using a $1 \mathrm{~L}$ multipurpose sprayer (Deluxe pressure sprayer), with a spray volume of $100 \mathrm{~mL} \mathrm{~m}^{-2}$ [24]. Randomized complete block design (RCBD) was set with four replications for the experiment. 


\subsubsection{Cultural Practices}

Watering was done manually using a watering can every day in the morning between 8.30 and $9.30 \mathrm{am}$. Unwanted weeds were cleared by manually using hands every five days.

\subsubsection{Data Collection}

\section{Plant Injury}

Visual assessments of plant injury were measured at 1,7,14, 21 days after herbicide treatments, and the growth response towards herbicide application was compared with respective untreated (control). The efficacy of the herbicides was measured by an injury scale (European System of Weed Control, and Crop Injury Evaluation) developed by Burrill et al. [25], where $0=$ no effect (all foliage green and alive), $>70 \%=$ adequate control, and $100 \%=$ complete kill $($ dead $)($ Table 1$)$.

Table 1. Injury rating scale.

\begin{tabular}{ccc}
\hline Scale & Injury (\%) & Effects on Weeds \\
\hline 1 & 0 & No effect (all foliage green and alive) \\
2 & $1-10$ & Very light symptoms (very minor chlorosis and/or leaf curling) \\
3 & $11-30$ & Light symptoms \\
4 & $31-49$ & Symptoms not reflected in the yield \\
5 & 50 & Medium (moderate chlorosis and/or leaf curling) \\
6 & $51-70$ & Heavy damage \\
7 & $71-90$ & Very heavy damage (severe chlorosis and/or dead leaves) \\
8 & $91-99$ & Complete kill (dead) \\
9 & 100 &
\end{tabular}

Fresh and Dry Weight

Weeds and crops were harvested at $1 \mathrm{~cm}$ above the ground level at 21 days after spray (DAS). Immediately the fresh weight was taken, and later, the samples were dried for $72 \mathrm{~h}$ at $65^{\circ} \mathrm{C}$ in the oven, and then the dry weight was taken. The fresh and dry weights were taken using a digital balance. The weed control efficiency treatment was calculated according to Abdullah et al. [26]:

Weed Control Efficiency $(\%)=\frac{\text { Dry weight of Untreated Tray }- \text { Dry Weight of Treated Tray }}{\text { Dry Weight of Untreated Tray }} \times 100$

The crop growth reduction was calculated for crop species and expressed as a percentage compared with the untreated (control) according to Motmainna et al. [24]:

Crop Growth Reduction $(\%)=\frac{\text { Dry weight of Untreated Tray }- \text { Dry Weight of Treated Tray }}{\text { Dry Weight of Untreated Tray }} \times 100$

\section{Plant Height}

Plant height was measured from the soil surface for all plant species using a measuring tape at 21 DAS.

\subsection{Field Trial Experiment}

\subsubsection{Experimental Site and Field Setup}

The weed control efficacy experiment was conducted in small plots, where typical weed communities were allowed to grow until uniform density across the plots was achieved. The trial was conducted from August to October 2020, at the Faculty of Agriculture in Farm 10, Universiti Putra Malaysia $\left(3^{\circ} 02^{\prime} \mathrm{N}\right.$ latitude and $101^{\circ} 42^{\prime}$ E longitude at $31 \mathrm{~m}$ above the sea level), Serdang, Selangor, Malaysia. The average maximum and minimum temperatures were 29.09 and $23.30^{\circ} \mathrm{C}$, respectively, with $85 \%$ relative humidity. Each plot size measured $2.5 \times 2.5 \mathrm{~m}$ and arranged in RCBD design with four replications. 


\subsubsection{Initial Vegetation Analysis}

Initial analysis of weed species was directed in the test plots before herbicide application, which helps to determine the weed composition based on their number, density, growth, and dominance in the experimental area. Weed species identification was done according to Bernes and Lus [27] and Fee et al. [28]. The classification of weed species and collection of samples followed the square method using 0.5 by $0.5 \mathrm{~m}$ quadrates. In each plot, a quadrat was randomly placed three times to collect the weed sample. Weed species present in the quadrates were identified, counted, clipped from ground level, and dried in the oven at $65^{\circ} \mathrm{C}$ for $72 \mathrm{~h}$.

\subsubsection{Herbicide Treatments}

The experimental plots were treated in a way to control weed species with different rates of WeedLock, glyphosate isopropyl-amine, and glufosinate-ammonium, including untreated (control). WeedLock was applied at 1345.50 and $2691.00 \mathrm{~L} \mathrm{ha}^{-1}$, while glyphosate isopropyl-amine (Roundup ${ }^{\circledR}$ ) and glufosinate-ammonium (Basta ${ }^{\circledR}$ ) were applied at 2 and $4 \mathrm{~L} \mathrm{ha}^{-1}$, respectively. The herbicides were sprayed using a flat-fan nozzle with a batteryoperated knapsack sprayer Matabi (Goizper, Gipuzkoa, Spain) delivering $450 \mathrm{~L}$ ha water at $200 \mathrm{kpa}$. Spray calibration was conducted before applying the herbicide treatments to determine the application rate, flow rate, spray width, and forward speed.

\subsubsection{Data Collection}

Dominance of Weed Species

The summed dominance ratio represented the dominance of weed species that infest the plots. The summed dominance ratio is calculated using the following formula described by Juraimi et al. [29] and Janiya and Moody [30]. Values are expressed as a percentage, as follows:

$$
\begin{array}{r}
\text { Summed Dominance Ratio }(\mathrm{SDR})=\frac{\text { Relative Density }+ \text { Relative Dry Weight }}{2} \\
\text { Relative Density }(\%)=\frac{\text { Density of a Particular Species }}{\text { Total Density }} \times 100 \\
\text { Relative Dry Weight }(\%)=\frac{\text { Dry Weight of a Particular Species }}{\text { Total Dry Weight }} \times 100
\end{array}
$$

Comparison of species affiliation and measurement of dominance among weed communities between treatments were made using the "Sorenson's Index of Similarity" [29,31]. Computation of the $S$ value is as follows:

$$
\mathrm{S}=\frac{2 \mathrm{~J}}{\mathrm{~A}+\mathrm{B}} \times 100
$$

where, $\mathrm{S}=$ Index of association between treatment $\mathrm{A}$ and $\mathrm{B} . \mathrm{J}=$ Number of species common in both treatment $A$ and $B . A=$ Number of species present in treatment $A . B=$ Number of species present in treatment $\mathrm{B}$.

\section{Evaluation of Treatment Efficacy}

The level of weed control was measured by the square method. Weed killed means all tissues of the observed plant were completely dead. At 1, 3, 7, 14, 21, 28, 35, and 60 DAS, weed control was determined by visual observation using an injury scale (Table 1).

\section{Fresh and Dry Weight}

Weeds were harvested aboveground at 35 and 60 DAS, and fresh weight was taken immediately. After that, the samples were dried for $72 \mathrm{~h}$ at $65^{\circ} \mathrm{C}$, and the dry weight was measured. The fresh and dry weights were taken using a digital balance. The weed control efficiency treatment was calculated according to Abdullah et al. [26], as follows: 
Weed Control Efficiency $(\%)=\frac{\text { Dry Weight of Untreated Plot }- \text { Dry Weight of Treated Plot }}{\text { Dry Weight of Untreated Plot }} \times 100$

\subsection{Statistical Analysis}

A two-way ANOVA (analysis of variance) was performed to find significant differences among each herbicide and the untreated (control) for both experiments. The differences among the treatment means were grouped by the Tukey test with 0.05 probability levels. The analysis was conducted by using SAS (statistical analysis system) software, version 9.4 (Cary, NC, USA).

\section{Results}

\subsection{Glasshouse Experiment}

3.1.1. Effects of WeedLock, Glyphosate Isopropyl-Amine, and Glufosinate-Ammonium on Visual Injury of Selected Weed Species

The efficacy of herbicides on A. conzyoides, E. hirta, A. compressus, E. indica, C. iria, and F. miliacea was assessed visually at $1,7,14,21$ DAS (Table 2). The results highlighted that the use of both WeedLock and chemical herbicides comparatively exhibited $(p \leq 0.05)$ the tested weeds over the untreated (control). One day after spray, WeedLock efficacy was significantly higher than glyphosate isopropyl-amine and glufosinate-ammonium in all tested weed species. Meanwhile, at 7 DAS, all treated weed species were completely killed or controlled $100 \%$ by the application of WeedLock at $1345.50\left(\mathrm{~T}_{2}\right)$ and $2691.00 \mathrm{~L} \mathrm{ha}^{-1}$ $\left(\mathrm{T}_{3}\right)$. Similar efficacy was also produced by glyphosate isopropyl-amine and glufosinateammonium at $2 \mathrm{~L} \mathrm{ha}^{-1}\left(\mathrm{~T}_{2}\right)$. At 21 DAS, a slightly different pattern was observed. The efficacy of WeedLock at $\mathrm{T}_{2}\left(1345.50 \mathrm{~L} \mathrm{ha}^{-1}\right.$ ) was similar (complete kill/dead) to glyphosate isopropyl-amine and glufosinate-ammonium in $\mathrm{T}_{2}\left(2 \mathrm{~L} \mathrm{ha}^{-1}\right)$ for all weeds except $E$. hirta and $A$. compressus. However, E. hirta and A. compressus exhibited heavy damage such as chlorosis and/or dead leaves and a rating of 7.75 on the visual assessment injury scale. On the contrary, WeedLock applied at a lower/half recommended rate $\left(\mathrm{T}_{1}\right)$ significantly reduced the efficacy at 21 DAS and produced lower weed control compared to glyphosate isopropyl-amine and glufosinate-ammonium. A. conyzoides, E. hirta, E. indica, A. compressus, C. iria, and F. miliacea were injured moderately at a lower rate $\left(\mathrm{T}_{1}\right)$ of WeedLock with an injury rating scale of $6.75,6.75,6.75,4.25,6.50$, and 4.50, respectively. Meanwhile, at 21 DAS, no significant difference in efficacy was observed between herbicides when sprayed at a higher rate $\left(\mathrm{T}_{3}\right)$, as all tested weeds were completely killed/died. The efficacy of WeedLock was similar to glyphosate isopropyl-amine and glufosinate-ammonium at the recommended rate $\left(T_{2}\right)$ and higher rate $\left(T_{3}\right)$; however, at a lower rate $\left(T_{1}\right)$, the efficacy of WeedLock was lower than synthetic herbicides (glyphosate isopropyl-amine and glufosinate-ammonium).

3.1.2. Effects of WeedLock, Glyphosate Isopropyl-Amine, and Glufosinate-Ammonium on Fresh Weight, Dry Weight, and Plant Height of Selected Weed Species

Growth traits, such as fresh weight, dry weight, and plant height, were recorded at 21 DAS, which were significantly $(p \leq 0.05)$ lower compared to the untreated (control) for all the rates of WeedLock, glyphosate isopropyl-amine, and glufosinate-ammonium (Table 3). A lower/half dose of WeedLock $\left(\mathrm{T}_{1}-672.75 \mathrm{~L} \mathrm{ha}^{-1}\right)$ showed $85.05 \%$ fresh weight reduction in A. conyzoides, $66.36 \%$ in E. hirta, $88.50 \%$ in E. indica, $76.20 \%$ in A. compressus, $85.65 \%$ in C. iria, and $56.92 \%$ in F. miliacea. The reduction in fresh weight ranged from $76.60 \%$ to $96.34 \%$ for bioherbicide WeedLock, $88.35 \%$ to $98.03 \%$ for glyphosate isopropyl-amine, and $80.77 \%$ to $97.20 \%$ for glufosinate-ammonium at the recommended rate $\left(\mathrm{T}_{2}\right)$. WeedLock exerted a similar effect with glyphosate isopropyl-amine and glufosinate-ammonium; for instance, at a higher dose (2691.00 L ha ${ }^{-1}$ ) of WeedLock, the fresh weight of $A$. conyzoides was reduced to $97.64 \%$, while $97.69 \%$ and $97.56 \%$ reduction was caused by glyphosate isopropyl-amine and glufosinate-ammonium, respectively, at $4 \mathrm{~L} \mathrm{ha}^{-1}$ compared to untreated (control). 
Table 2. Effects of WeedLock, glyphosate isopropyl-amine, and glufosinate-ammonium on an injury scale of tested weed species.

\begin{tabular}{|c|c|c|c|c|c|c|c|c|c|c|c|c|c|}
\hline \multirow{3}{*}{ Test Crops } & \multirow{3}{*}{ Doses } & \multicolumn{12}{|c|}{ Injury Scale } \\
\hline & & \multicolumn{3}{|c|}{1 DAS } & \multicolumn{3}{|c|}{7 DAS } & \multicolumn{3}{|c|}{14 DAS } & \multicolumn{3}{|c|}{21 DAS } \\
\hline & & WL & GLY & GLU & WL & GLY & GLU & WL & GLY & GLU & WL & GLY & GLU \\
\hline $\begin{array}{l}\text { Ageratum } \\
\text { conyzoides }\end{array}$ & $\begin{array}{l}\mathrm{T}_{0} \\
\mathrm{~T}_{1} \\
\mathrm{~T}_{2} \\
\mathrm{~T}_{3}\end{array}$ & $\begin{array}{c}1.00 \mathrm{a} \pm 0 \\
7.25 \mathrm{a} \pm 0.25 \\
8.00 \mathrm{a} \pm 0 \\
8.00 \mathrm{a} \pm 0\end{array}$ & $\begin{array}{l}1.00 \mathrm{a} \pm 0 \\
2.00 \mathrm{~b} \pm 0 \\
2.00 \mathrm{~b} \pm 0 \\
2.00 \mathrm{c} \pm 0\end{array}$ & $\begin{array}{c}1.00 \mathrm{a} \pm 0 \\
2.00 \mathrm{~b} \pm 0 \\
2.50 \mathrm{~b} \pm 0.29 \\
2.75 \mathrm{~b} \pm 0.25\end{array}$ & $\begin{array}{c}1.00 \mathrm{a} \pm 0 \\
6.75 \mathrm{~b} \pm 0.25 \\
8.25 \mathrm{a} \pm 0.48 \\
9.00 \mathrm{a} \pm 0\end{array}$ & $\begin{array}{l}1.00 \mathrm{a} \pm 0 \\
9.00 \mathrm{a} \pm 0 \\
9.00 \mathrm{a} \pm 0 \\
9.00 \mathrm{a} \pm 0\end{array}$ & $\begin{array}{l}1.00 \mathrm{a} \pm 0 \\
9.00 \mathrm{a} \pm 0 \\
9.00 \mathrm{a} \pm 0 \\
9.00 \mathrm{a} \pm 0\end{array}$ & $\begin{array}{c}1.00 \mathrm{a} \pm 0 \\
6.75 \mathrm{~b} \pm 0.25 \\
8.25 \mathrm{a} \pm 0.48 \\
9.00 \mathrm{a} \pm 0\end{array}$ & $\begin{array}{l}1.00 \mathrm{a} \pm 0 \\
9.00 \mathrm{a} \pm 0 \\
9.00 \mathrm{a} \pm 0 \\
9.00 \mathrm{a} \pm 0\end{array}$ & $\begin{array}{l}1.00 \mathrm{a} \pm 0 \\
9.00 \mathrm{a} \pm 0 \\
9.00 \mathrm{a} \pm 0 \\
9.00 \mathrm{a} \pm 0\end{array}$ & $\begin{array}{c}1.00 \mathrm{a} \pm 0 \\
6.75 \mathrm{~b} \pm 0.25 \\
9.00 \mathrm{a} \pm 0 \\
9.00 \mathrm{a} \pm 0\end{array}$ & $\begin{array}{l}1.00 \mathrm{a} \pm 0 \\
9.00 \mathrm{a} \pm 0 \\
9.00 \mathrm{a} \pm 0 \\
9.00 \mathrm{a} \pm 0\end{array}$ & $\begin{array}{l}1.00 \mathrm{a} \pm 0 \\
9.00 \mathrm{a} \pm 0 \\
9.00 \mathrm{a} \pm 0 \\
9.00 \mathrm{a} \pm 0\end{array}$ \\
\hline Euphorbia hirta & $\begin{array}{l}\mathrm{T}_{0} \\
\mathrm{~T}_{1} \\
\mathrm{~T}_{2} \\
\mathrm{~T}_{3}\end{array}$ & $\begin{array}{c}1.00 \mathrm{a} \pm 0 \\
7.25 \mathrm{a} \pm 0.25 \\
8.00 \mathrm{a} \pm 0 \\
8.00 \mathrm{a} \pm 0\end{array}$ & $\begin{array}{l}1.00 \mathrm{a} \pm 0 \\
2.00 \mathrm{~b} \pm 0 \\
2.00 \mathrm{~b} \pm 0 \\
2.00 \mathrm{~b} \pm 0\end{array}$ & $\begin{array}{c}1.00 \mathrm{a} \pm 0 \\
2.00 \mathrm{~b} \pm 0 \\
2.50 \mathrm{~b} \pm 0.29 \\
2.50 \mathrm{~b} \pm 0.29\end{array}$ & $\begin{array}{c}1.00 \mathrm{a} \pm 0 \\
6.50 \mathrm{~b} \pm 0.29 \\
7.75 \mathrm{~b} \pm 0.48 \\
9.00 \mathrm{a} \pm 0\end{array}$ & $\begin{array}{l}1.00 \mathrm{a} \pm 0 \\
9.00 \mathrm{a} \pm 0 \\
9.00 \mathrm{a} \pm 0 \\
9.00 \mathrm{a} \pm 0\end{array}$ & $\begin{array}{l}1.00 \mathrm{a} \pm 0 \\
9.00 \mathrm{a} \pm 0 \\
9.00 \mathrm{a} \pm 0 \\
9.00 \mathrm{a} \pm 0\end{array}$ & $\begin{array}{c}1.00 \mathrm{a} \pm 0 \\
6.75 \mathrm{~b} \pm 0.25 \\
7.75 \mathrm{~b} \pm 0.48 \\
9.00 \mathrm{a} \pm 0\end{array}$ & $\begin{array}{l}1.00 \mathrm{a} \pm 0 \\
9.00 \mathrm{a} \pm 0 \\
9.00 \mathrm{a} \pm 0 \\
9.00 \mathrm{a} \pm 0\end{array}$ & $\begin{array}{l}1.00 \mathrm{a} \pm 0 \\
9.00 \mathrm{a} \pm 0 \\
9.00 \mathrm{a} \pm 0 \\
9.00 \mathrm{a} \pm 0\end{array}$ & $\begin{array}{c}1.00 \mathrm{a} \pm 0 \\
6.75 \mathrm{~b} \pm 0.25 \\
7.75 \mathrm{~b} \pm 0.48 \\
9.00 \mathrm{a} \pm 0\end{array}$ & $\begin{array}{l}1.00 \mathrm{a} \pm 0 \\
9.00 \mathrm{a} \pm 0 \\
9.00 \mathrm{a} \pm 0 \\
9.00 \mathrm{a} \pm 0\end{array}$ & $\begin{array}{l}1.00 \mathrm{a} \pm 0 \\
9.00 \mathrm{a} \pm 0 \\
9.00 \mathrm{a} \pm 0 \\
9.00 \mathrm{a} \pm 0\end{array}$ \\
\hline Eleusine indica & $\begin{array}{l}\mathrm{T}_{0} \\
\mathrm{~T}_{1} \\
\mathrm{~T}_{2} \\
\mathrm{~T}_{3} \\
\end{array}$ & $\begin{array}{c}1.00 \mathrm{a} \pm 0 \\
7.50 \mathrm{a} \pm 0.29 \\
8.00 \mathrm{a} \pm 0 \\
8.00 \mathrm{a} \pm 0 \\
\end{array}$ & $\begin{array}{l}1.00 \mathrm{a} \pm 0 \\
2.00 \mathrm{~b} \pm 0 \\
2.00 \mathrm{~b} \pm 0 \\
2.00 \mathrm{c} \pm 0 \\
\end{array}$ & $\begin{array}{c}1.00 \mathrm{a} \pm 0 \\
2.00 \mathrm{~b} \pm 0 \\
2.50 \mathrm{~b} \pm 0.29 \\
2.75 \mathrm{~b} \pm 0.25 \\
\end{array}$ & $\begin{array}{c}1.00 \mathrm{a} \pm 0 \\
7.00 \mathrm{~b} \pm 0.41 \\
9.00 \mathrm{a} \pm 0 \\
9.00 \mathrm{a} \pm 0 \\
\end{array}$ & $\begin{array}{l}1.00 \mathrm{a} \pm 0 \\
9.00 \mathrm{a} \pm 0 \\
9.00 \mathrm{a} \pm 0 \\
9.00 \mathrm{a} \pm 0\end{array}$ & $\begin{array}{l}1.00 \mathrm{a} \pm 0 \\
9.00 \mathrm{a} \pm 0 \\
9.00 \mathrm{a} \pm 0 \\
9.00 \mathrm{a} \pm 0\end{array}$ & $\begin{array}{c}1.00 \mathrm{a} \pm 0 \\
6.75 \mathrm{~b} \pm 0.48 \\
9.00 \mathrm{a} \pm 0 \\
9.00 \mathrm{a} \pm 0 \\
\end{array}$ & $\begin{array}{l}1.00 \mathrm{a} \pm 0 \\
9.00 \mathrm{a} \pm 0 \\
9.00 \mathrm{a} \pm 0 \\
9.00 \mathrm{a} \pm 0\end{array}$ & $\begin{array}{l}1.00 \mathrm{a} \pm 0 \\
9.00 \mathrm{a} \pm 0 \\
9.00 \mathrm{a} \pm 0 \\
9.00 \mathrm{a} \pm 0\end{array}$ & $\begin{array}{c}1.00 \mathrm{a} \pm 0 \\
6.75 \mathrm{~b} \pm 0.48 \\
9.00 \mathrm{a} \pm 0 \\
9.00 \mathrm{a} \pm 0 \\
\end{array}$ & $\begin{array}{l}1.00 \mathrm{a} \pm 0 \\
9.00 \mathrm{a} \pm 0 \\
9.00 \mathrm{a} \pm 0 \\
9.00 \mathrm{a} \pm 0\end{array}$ & $\begin{array}{l}1.00 \mathrm{a} \pm 0 \\
9.00 \mathrm{a} \pm 0 \\
9.00 \mathrm{a} \pm 0 \\
9.00 \mathrm{a} \pm 0\end{array}$ \\
\hline $\begin{array}{l}\text { Axonopus } \\
\text { compressus }\end{array}$ & $\begin{array}{l}\mathrm{T}_{0} \\
\mathrm{~T}_{1} \\
\mathrm{~T}_{2} \\
\mathrm{~T}_{3} \\
\end{array}$ & $\begin{array}{c}1.00 \mathrm{a} \pm 0 \\
4.75 \mathrm{a} \pm 0.48 \\
7.75 \mathrm{a} \pm 0.25 \\
8.00 \mathrm{a} \pm 0\end{array}$ & $\begin{array}{l}1.00 \mathrm{a} \pm 0 \\
2.00 \mathrm{~b} \pm 0 \\
2.00 \mathrm{~b} \pm 0 \\
2.00 \mathrm{~b} \pm 0\end{array}$ & $\begin{array}{c}1.00 \mathrm{a} \pm 0 \\
2.00 \mathrm{~b} \pm 0 \\
2.50 \mathrm{~b} \pm 0.29 \\
2.50 \mathrm{~b} \pm 0.29 \\
\end{array}$ & $\begin{array}{c}1.00 \mathrm{a} \pm 0 \\
4.50 \mathrm{~b} \pm 0.64 \\
7.75 \mathrm{~b} \pm 0.25 \\
9.00 \mathrm{a} \pm 0\end{array}$ & $\begin{array}{l}1.00 \mathrm{a} \pm 0 \\
9.00 \mathrm{a} \pm 0 \\
9.00 \mathrm{a} \pm 0 \\
9.00 \mathrm{a} \pm 0\end{array}$ & $\begin{array}{l}1.00 \mathrm{a} \pm 0 \\
9.00 \mathrm{a} \pm 0 \\
9.00 \mathrm{a} \pm 0 \\
9.00 \mathrm{a} \pm 0\end{array}$ & $\begin{array}{c}1.00 \mathrm{a} \pm 0 \\
4.25 \mathrm{~b} \pm 0.48 \\
7.75 \mathrm{~b} \pm 0.25 \\
9.00 \mathrm{a} \pm 0\end{array}$ & $\begin{array}{l}1.00 \mathrm{a} \pm 0 \\
9.00 \mathrm{a} \pm 0 \\
9.00 \mathrm{a} \pm 0 \\
9.00 \mathrm{a} \pm 0\end{array}$ & $\begin{array}{l}1.00 \mathrm{a} \pm 0 \\
9.00 \mathrm{a} \pm 0 \\
9.00 \mathrm{a} \pm 0 \\
9.00 \mathrm{a} \pm 0\end{array}$ & $\begin{array}{c}1.00 \mathrm{a} \pm 0 \\
4.25 \mathrm{~b} \pm 0.48 \\
7.75 \mathrm{~b} \pm 0.25 \\
9.00 \mathrm{a} \pm 0\end{array}$ & $\begin{array}{l}1.00 \mathrm{a} \pm 0 \\
9.00 \mathrm{a} \pm 0 \\
9.00 \mathrm{a} \pm 0 \\
9.00 \mathrm{a} \pm 0\end{array}$ & $\begin{array}{l}1.00 \mathrm{a} \pm 0 \\
9.00 \mathrm{a} \pm 0 \\
9.00 \mathrm{a} \pm 0 \\
9.00 \mathrm{a} \pm 0\end{array}$ \\
\hline Cyperus iria & $\begin{array}{l}\mathrm{T}_{0} \\
\mathrm{~T}_{1} \\
\mathrm{~T}_{2} \\
\mathrm{~T}_{3} \\
\end{array}$ & $\begin{array}{c}1.00 \mathrm{a} \pm 0 \\
7.00 \mathrm{a} \pm 0.41 \\
8.00 \mathrm{a} \pm 0 \\
8.00 \mathrm{a} \pm 0\end{array}$ & $\begin{array}{l}1.00 \mathrm{a} \pm 0 \\
2.00 \mathrm{~b} \pm 0 \\
2.00 \mathrm{~b} \pm 0 \\
2.00 \mathrm{~b} \pm 0\end{array}$ & $\begin{array}{c}1.00 \mathrm{a} \pm 0 \\
2.00 \mathrm{~b} \pm 0 \\
2.50 \mathrm{~b} \pm 0.29 \\
2.50 \mathrm{~b} \pm 0.29 \\
\end{array}$ & $\begin{array}{c}1.00 \mathrm{a} \pm 0 \\
6.50 \mathrm{~b} \pm 0.29 \\
9.00 \mathrm{a} \pm 0 \\
9.00 \mathrm{a} \pm 0\end{array}$ & $\begin{array}{l}1.00 \mathrm{a} \pm 0 \\
9.00 \mathrm{a} \pm 0 \\
9.00 \mathrm{a} \pm 0 \\
9.00 \mathrm{a} \pm 0\end{array}$ & $\begin{array}{l}1.00 \mathrm{a} \pm 0 \\
9.00 \mathrm{a} \pm 0 \\
9.00 \mathrm{a} \pm 0 \\
9.00 \mathrm{a} \pm 0\end{array}$ & $\begin{array}{c}1.00 \mathrm{a} \pm 0 \\
6.50 \mathrm{~b} \pm 0.29 \\
9.00 \mathrm{a} \pm 0 \\
9.00 \mathrm{a} \pm 0\end{array}$ & $\begin{array}{l}1.00 \mathrm{a} \pm 0 \\
9.00 \mathrm{a} \pm 0 \\
9.00 \mathrm{a} \pm 0 \\
9.00 \mathrm{a} \pm 0\end{array}$ & $\begin{array}{l}1.00 \mathrm{a} \pm 0 \\
9.00 \mathrm{a} \pm 0 \\
9.00 \mathrm{a} \pm 0 \\
9.00 \mathrm{a} \pm 0\end{array}$ & $\begin{array}{c}1.00 \mathrm{a} \pm 0.0 \\
6.50 \mathrm{~b} \pm 0.29 \\
9.00 \mathrm{a} \pm 0 \\
9.00 \mathrm{a} \pm 0\end{array}$ & $\begin{array}{l}1.00 \mathrm{~b} \pm 0 \\
9.00 \mathrm{a} \pm 0 \\
9.00 \mathrm{a} \pm 0 \\
9.00 \mathrm{a} \pm 0\end{array}$ & $\begin{array}{l}1.00 \mathrm{~b} \pm 0 \\
9.00 \mathrm{a} \pm 0 \\
9.00 \mathrm{a} \pm 0 \\
9.00 \mathrm{a} \pm 0\end{array}$ \\
\hline $\begin{array}{c}\text { Fimbristylis } \\
\text { miliacea }\end{array}$ & $\begin{array}{l}\mathrm{T}_{0} \\
\mathrm{~T}_{1} \\
\mathrm{~T}_{2} \\
\mathrm{~T}_{3}\end{array}$ & $\begin{array}{c}1.00 \mathrm{a} \pm 0 \\
5.25 \mathrm{a} \pm 0.48 \\
7.50 \mathrm{a} \pm 0.29 \\
8.00 \mathrm{a} \pm 0\end{array}$ & $\begin{array}{l}1.00 \mathrm{a} \pm 0 \\
2.00 \mathrm{~b} \pm 0 \\
2.00 \mathrm{~b} \pm 0 \\
2.00 \mathrm{~b} \pm 0\end{array}$ & $\begin{array}{c}1.00 \mathrm{a} \pm 0 \\
2.00 \mathrm{~b} \pm 0 \\
2.50 \mathrm{~b} \pm 0.29 \\
2.50 \mathrm{~b} \pm 0.29\end{array}$ & $\begin{array}{c}1.00 \mathrm{a} \pm 0 \\
4.50 \mathrm{~b} \pm 0.64 \\
8.50 \mathrm{a} \pm 0.29 \\
9.00 \mathrm{a} \pm 0\end{array}$ & $\begin{array}{l}1.00 \mathrm{a} \pm 0 \\
9.00 \mathrm{a} \pm 0 \\
9.00 \mathrm{a} \pm 0 \\
9.00 \mathrm{a} \pm 0\end{array}$ & $\begin{array}{l}1.00 \mathrm{a} \pm 0 \\
9.00 \mathrm{a} \pm 0 \\
9.00 \mathrm{a} \pm 0 \\
9.00 \mathrm{a} \pm 0\end{array}$ & $\begin{array}{c}1.00 \mathrm{a} \pm 0 \\
4.50 \mathrm{~b} \pm 0.64 \\
8.50 \mathrm{a} \pm 0.29 \\
9.00 \mathrm{a} \pm 0\end{array}$ & $\begin{array}{l}1.00 \mathrm{a} \pm 0 \\
9.00 \mathrm{a} \pm 0 \\
9.00 \mathrm{a} \pm 0 \\
9.00 \mathrm{a} \pm 0\end{array}$ & $\begin{array}{l}1.00 \mathrm{a} \pm 0 \\
9.00 \mathrm{a} \pm 0 \\
9.00 \mathrm{a} \pm 0 \\
9.00 \mathrm{a} \pm 0\end{array}$ & $\begin{array}{c}1.00 \mathrm{a} \pm 0 \\
4.50 \mathrm{~b} \pm 0.64 \\
8.50 \mathrm{a} \pm 0.29 \\
9.00 \mathrm{a} \pm 0\end{array}$ & $\begin{array}{l}1.00 \mathrm{a} \pm 0 \\
9.00 \mathrm{a} \pm 0 \\
9.00 \mathrm{a} \pm 0 \\
9.00 \mathrm{a} \pm 0\end{array}$ & $\begin{array}{l}1.00 \mathrm{a} \pm 0 \\
9.00 \mathrm{a} \pm 0 \\
9.00 \mathrm{a} \pm 0 \\
9.00 \mathrm{a} \pm 0\end{array}$ \\
\hline
\end{tabular}

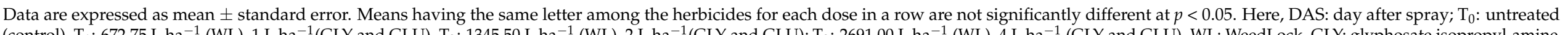

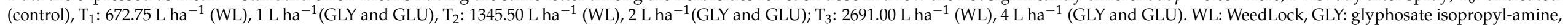

GLU: glufosinate-ammonium. 


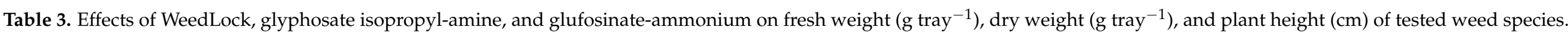

\begin{tabular}{|c|c|c|c|c|c|c|c|c|c|c|}
\hline \multirow{2}{*}{ Test Weeds } & \multirow{2}{*}{ Doses } & \multicolumn{3}{|c|}{ Fresh Weight (g) } & \multicolumn{3}{|c|}{ Dry Weight (g) } & \multicolumn{3}{|c|}{ Plant Height (cm) } \\
\hline & & WL & GLY & GLU & WL & GLY & GLU & WL & GLY & GLU \\
\hline \multirow{4}{*}{$\begin{array}{l}\text { Ageratum } \\
\text { conyzoides }\end{array}$} & $\mathrm{T}_{0}$ & $35.68 \mathrm{a} \pm 0.93$ & $36.21 \mathrm{a} \pm 0.66$ & $35.88 \mathrm{a} \pm 0.72$ & $8.62 \mathrm{a} \pm 0.15$ & $8.44 a \pm 0.11$ & $8.26 a \pm 0.06$ & $43.3 a \pm 0.52$ & $41.0 \mathrm{~b} \pm 0.65$ & $41.2 b \pm 0.74$ \\
\hline & $\mathrm{T}_{1}$ & $5.30 \mathrm{a} \pm 0.49$ & $1.91 b \pm 0.11$ & $2.07 \mathrm{~b} \pm 0.47$ & $0.80 \mathrm{a} \pm 0.07$ & $0.77 \mathrm{a} \pm 0.07$ & $0.90 \mathrm{a} \pm 0.07$ & $21.3 \mathrm{a} \pm 0.84$ & $0.00 \mathrm{a} \pm 0$ & $0.00 \mathrm{~b} \pm 0$ \\
\hline & $\mathrm{T}_{2}$ & $1.93 a \pm 0.32$ & $0.99 b \pm 0.09$ & $1.29 \mathrm{ab} \pm 0.13$ & $0.21 \mathrm{a} \pm 0.03$ & $0.20 \mathrm{a} \pm 0.03$ & $0.23 a \pm 0.03$ & $4.27 \mathrm{a} \pm 2.49$ & $0.00 \mathrm{a} \pm 0$ & $0.00 \mathrm{a} \pm 0$ \\
\hline & $\mathrm{T}_{3}$ & $0.85 a \pm 0.08$ & $0.84 a \pm 0.07$ & $0.88 \mathrm{a} \pm 0.08$ & $0.13 a \pm 0.01$ & $0.12 \mathrm{a} \pm 0.01$ & $0.15 a \pm 0.01$ & $0.00 \mathrm{a} \pm 0$ & $0.00 \mathrm{a} \pm 0$ & $0.00 \mathrm{a} \pm 0$ \\
\hline \multirow{3}{*}{ Euphorbia hirta } & $\mathrm{T}_{0}$ & $42.00 \mathrm{a} \pm 0.30$ & $40.20 \mathrm{~b} \pm 0.62$ & $40.88 \mathrm{ab} \pm 0.51$ & $12.13 a \pm 0.32$ & $11.69 \mathrm{a} \pm 0.25$ & $11.84 \mathrm{a} \pm 0.11$ & $30.66 \mathrm{a} \pm 0.56$ & $29.79 a \pm 0.33$ & $30.77 a \pm 0.37$ \\
\hline & $\mathrm{T}_{1}$ & $14.02 a \pm 0.64$ & $11.81 b \pm 0.42$ & $12.10 b \pm 0.29$ & $1.84 \mathrm{a} \pm 0.06$ & $1.18 b \pm 0.05$ & $1.26 \mathrm{~b} \pm 0.06$ & $17.19 a \pm 0.30$ & $0.00 \mathrm{~b} \pm 0$ & $0.00 \mathrm{~b} \pm 0$ \\
\hline & $\mathrm{T}_{3}$ & $3.89 a \pm 0.16$ & $1.74 b \pm 0.16$ & $2.06 \mathrm{~b} \pm 0.22$ & $0.95 a \pm 0.04$ & $0.35 b \pm 0.05$ & $0.45 b \pm 0.06$ & $0.00 \mathrm{a} \pm 0$ & $0.00 \mathrm{a} \pm 0$ & $0.00 \mathrm{a} \pm 0$ \\
\hline \multirow{4}{*}{ Eleusine indica } & $\mathrm{T}_{0}$ & $79.51 \mathrm{a} \pm 0.29$ & $80.51 \mathrm{a} \pm 0.50$ & $80.30 \mathrm{a} \pm 0.32$ & $19.75 \mathrm{a} \pm 0.46$ & $19.05 \mathrm{a} \pm 0.51$ & $19.35 a \pm 0.14$ & $71.47 \mathrm{a} \pm 0.31$ & $71.88 \mathrm{a} \pm 0.39$ & $70.56 a \pm 0.64$ \\
\hline & $\mathrm{T}_{1}$ & $9.14 a \pm 0.62$ & $6.87 \mathrm{~b} \pm 0.28$ & $7.69 b \pm 0.22$ & $1.88 \mathrm{a} \pm 0.12$ & $1.49 a \pm 0.18$ & $1.61 \mathrm{a} \pm 0.10$ & $30.68 \mathrm{a} \pm 0.88$ & $0.00 \mathrm{~b} \pm 0$ & $0.00 \mathrm{~b} \pm 0$ \\
\hline & $\mathrm{T}_{2}$ & $2.91 \mathrm{a} \pm 0.11$ & $1.58 b \pm 0.11$ & $2.25 c \pm 0.12$ & $0.52 b \pm 0.05$ & $0.63 \mathrm{ab} \pm 0.04$ & $0.70 \mathrm{a} \pm 0.05$ & $0.00 \mathrm{a} \pm 0$ & $0.00 \mathrm{a} \pm 0$ & $0.00 \mathrm{a} \pm 0$ \\
\hline & $\mathrm{T}_{3}$ & $1.73 a \pm 0.11$ & $1.24 b \pm 0.06$ & $1.45 \mathrm{ab} \pm 0.10$ & $0.33 b \pm 0.02$ & $0.30 \mathrm{~b} \pm 0.03$ & $0.49 a \pm 0.06$ & $0.00 \mathrm{a} \pm 0$ & $0.00 \mathrm{a} \pm 0$ & $0.00 \mathrm{a} \pm 0$ \\
\hline \multirow{3}{*}{$\begin{array}{l}\text { Axonopus } \\
\text { compressus }\end{array}$} & $\mathrm{T}_{0}$ & $66.14 \mathrm{a} \pm 0.61$ & $64.37 \mathrm{~b} \pm 0.40$ & $64.96 \mathrm{ab} \pm 0.39$ & $14.21 \mathrm{a} \pm 0.13$ & $13.77 \mathrm{a} \pm 0.23$ & $13.94 a \pm 0.21$ & $34.36 a \pm 0.46$ & $34.05 \mathrm{a} \pm 0.45$ & $34.40 \mathrm{a} \pm 0.28$ \\
\hline & $\mathrm{T}_{1}$ & $15.74 \mathrm{a} \pm 0.38$ & $4.78 b \pm 0.16$ & $5.02 b \pm 0.27$ & $2.36 a \pm 0.11$ & $1.31 b \pm 0.05$ & $1.11 b \pm 0.06$ & $13.87 a \pm 0.46$ & $0.00 \mathrm{~b} \pm 0$ & $0.00 \mathrm{~b} \pm 0$ \\
\hline & $\mathrm{T}_{2}$ & $6.72 a \pm 0.33$ & $2.08 b \pm 0.06$ & $2.23 b \pm 0.17$ & $1.29 \mathrm{a} \pm 0.06$ & $0.78 b \pm 0.07$ & $1.09 a \pm 0.07$ & $5.82 \mathrm{a} \pm 0.52$ & $0.00 \mathrm{a} \pm 0$ & $0.00 \mathrm{a} \pm 0$ \\
\hline \multirow{4}{*}{ Cyperus iria } & $\mathrm{T}_{0}$ & $45.22 b \pm 0.53$ & $47.16 a \pm 0.25$ & $47.03 a \pm 0.71$ & $8.72 a \pm 0.31$ & $9.17 a \pm 0.26$ & $9.13 a \pm 0.09$ & $54.88 \mathrm{a} \pm 0.76$ & $56.59 a \pm 0.72$ & $55.80 \mathrm{a} \pm 0.56$ \\
\hline & $\mathrm{T}_{1}$ & $6.48 a \pm 0.27$ & $2.32 c \pm 0.17$ & $3.11 b \pm 0.12$ & $1.29 \mathrm{a} \pm 0.15$ & $0.58 b \pm 0.04$ & $1.15 \mathrm{a} \pm 0.03$ & $21.65 \mathrm{a} \pm 1.05$ & $0.00 \mathrm{~b} \pm 0$ & $0.00 \mathrm{~b} \pm 0$ \\
\hline & $\mathrm{T}_{2}$ & $2.37 \mathrm{a} \pm 0.25$ & $1.39 b \pm 0.05$ & $2.26 a \pm 0.12$ & $0.43 \mathrm{ab} \pm 0.05$ & $0.33 b \pm 0.04$ & $0.68 \mathrm{a} \pm 0.14$ & $0.00 \mathrm{a} \pm 0$ & $0.00 \mathrm{a} \pm 0$ & $0.00 \mathrm{a} \pm 0$ \\
\hline & $\mathrm{T}_{3}$ & $1.45 a \pm 0.09$ & $1.01 \mathrm{~b} \pm 0.06$ & $1.37 \mathrm{a} \pm 0.08$ & $0.16 b \pm 0.02$ & $0.15 b \pm 0.02$ & $0.33 a \pm 0.04$ & $0.00 \mathrm{a} \pm 0$ & $0.00 \mathrm{a} \pm 0$ & $0.00 \mathrm{a} \pm 0$ \\
\hline \multirow{4}{*}{$\begin{array}{c}\text { Fimbristylis } \\
\text { miliacea }\end{array}$} & $\mathrm{T}_{0}$ & $24.74 b \pm 0.44$ & $25.76 \mathrm{ab} \pm 0.31$ & $25.95 a \pm 0.27$ & $3.48 \mathrm{a} \pm 0.07$ & $3.52 \mathrm{a} \pm 0.05$ & $3.55 \mathrm{a} \pm 0.04$ & $46.48 \mathrm{a} \pm 0.79$ & $47.56 a \pm 0.49$ & $47.65 a \pm 0.49$ \\
\hline & $\mathrm{T}_{1}$ & $10.63 a \pm 0.61$ & $4.36 b \pm 0.23$ & $4.43 b \pm 0.30$ & $0.74 \mathrm{a} \pm 0.05$ & $0.51 b \pm 0.06$ & $0.52 \mathrm{ab} \pm 0.09$ & $29.05 a \pm 0.48$ & $0.00 \mathrm{~b} \pm 0$ & $0.00 \mathrm{~b} \pm 0$ \\
\hline & $\mathrm{T}_{2}$ & $4.04 \mathrm{a} \pm 0.21$ & $2.43 b \pm 0.18$ & $2.53 b \pm 0.27$ & $0.57 \mathrm{a} \pm 0.03$ & $0.37 \mathrm{~b} \pm 0.05$ & $0.38 b \pm 0.05$ & $4.30 \mathrm{a} \pm 2.48$ & $0.00 \mathrm{a} \pm 0$ & $0.00 \mathrm{a} \pm 0$ \\
\hline & $\mathrm{T}_{3}$ & $1.92 \mathrm{a} \pm 0.08$ & $1.27 \mathrm{~b} \pm 0.11$ & $1.30 \mathrm{~b} \pm 0.14$ & $0.28 \mathrm{a} \pm 0.05$ & $0.20 \mathrm{a} \pm 0.04$ & $0.27 \mathrm{a} \pm 0.04$ & $0.00 \mathrm{a} \pm 0$ & $0.00 \mathrm{a} \pm 0$ & $0.00 \mathrm{a} \pm 0$ \\
\hline
\end{tabular}

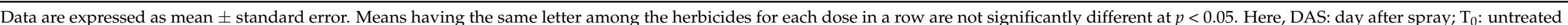

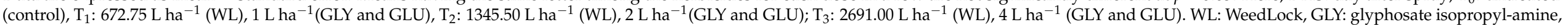

GLU: glufosinate-ammonium. 
Dry weights of all tested weeds responded differently to WeedLock, glyphosate isopropyl-amine, and glufosinate-ammonium (Table 3). WeedLock inhibited the dry weight of more than $90 \%$ for all tested weeds except $E$. hirta $(89.05 \%)$ and F. miliacea $(83.82 \%)$ in $\mathrm{T}_{2}$ $\left(1345.50 \mathrm{~L} \mathrm{ha}^{-1}\right)$. The dry weights of E. hirta, A. compressus, and C. iria were significantly ( $p$ $\leq 0.05)$ different; however, no significant $(p>0.05)$ difference was found in A. conzyoide, E. indica, and F. miliacea when WeedLock, glyphosate isopropyl-amine, and glufosinateammonium were applied at $672.75,1$, and $1 \mathrm{~L} \mathrm{ha}^{-1}$ respectively. A. conyzoides showed a $90.66 \%$ dry weight reduction while E. indica and F. miliacea showed $90.43 \%$ and $78.70 \%$, respectively, by WeedLock at a lower/half dose $\mathrm{T}_{1}\left(672.75 \mathrm{~L} \mathrm{ha}^{-1}\right)$. On the other hand, dry weight reduction by more than $90 \%$ occurred in all herbicides.

Plant heights of all tested weeds were significantly $(p \leq 0.05)$ influenced by WeedLock in a dose-dependent pattern compared to glyphosate isopropyl-amine and glufosinateammonium (Table 3). The untreated (control) plants obtained the highest plant height. However, plant height reduction varied among the tested weeds and the herbicides treatment. WeedLock reduced the plant height of all tested weeds from $37.42 \%$ to $60.55 \%$ at a lower dose $\left(\mathrm{T}_{1}-672.75 \mathrm{~L} \mathrm{ha}^{-1}\right)$ compared to untreated (control). At a lower dose of WeedLock $\left(672.75 \mathrm{~L} \mathrm{ha}^{-1}\right)$, C. iria exhibited the highest plant height reduction of $60.55 \%$, followed by $59.64 \%$ and $57.05 \%$ for A. compressus and E. indica, respectively. The highest reduction $(100 \%)$ occurred in E. indica and C. iria by WeedLock in $\mathrm{T}_{2}\left(1345.50 \mathrm{~L} \mathrm{ha}^{-1}\right)$. At a higher dose $\left(T_{3}\right)$, all herbicides exhibited the highest reduction $(100 \%)$ for all tested weed species when compared to untreated (control).

3.1.3. Effects of WeedLock, Glyphosate Isopropyl-Amine, and Glufosinate-Ammonium on Weed Control Efficiency of Selected Weed Species

WeedLock, glyphosate isopropyl-amine, and glufosinate-ammonium had a significant $(p \leq 0.05)$ influence on weed control efficiency of all test weed species, as presented in Table 4. WeedLock showed $90.66 \%$ to $98.49 \%$ control efficacy on A. conyzoides, $90.43 \%$ to $98.29 \%$ on E. indica, and $85.21 \%$ to $98.20 \%$ on C. iria, the lowest $\left(\mathrm{T}_{1}\right)$ to the highest $\left(\mathrm{T}_{3}\right)$ dose, respectively. Fimbristylis milieace was least controlled $(78.80 \%)$ by WeedLock in $\mathrm{T}_{1}\left(672.75 \mathrm{~L} \mathrm{ha}^{-1}\right)$ compared to other examined weeds. More than $97 \%$ weed control efficiency of WeedLock (1345.50 L ha $\left.{ }^{-1}\right)$, glyphosate isopropyl-amine $\left(2 \mathrm{~L} \mathrm{ha}^{-1}\right)$, and glufosinate-ammonium $\left(2 \mathrm{~L} \mathrm{ha}^{-1}\right)$ was recorded for $A$. conyzoides. Among the tested weeds, the weed control efficiency of WeedLock was higher on A. conyzoides at a higher dose of $\mathrm{T}_{3}$ (2691.00 $\mathrm{L} \mathrm{ha}^{-1}$ ) with an inhibition index of $98.49 \%$, followed by $98.29 \%, 98.20 \%, 97.07 \%$, $92.12 \%$, and $91.87 \%$ in E. indica, C. iria, A. compressus, E. hirta, and F. miliacea respectively. Similar weed control efficiency was observed on examined weed species when glyphosate isopropyl-amine and glufosinate-ammonium were applied at their highest dose in $\mathrm{T}_{3}$ $\left(4 \mathrm{~L} \mathrm{ha}^{-1}\right)$.

3.1.4. Effects of WeedLock, Glyphosate Isopropyl-Amine, and Glufosinate-Ammonium on Injury Scale of Selected Crop Species

The phytotoxic effects of WeedLock, glyphosate isopropyl-amine, and glufosinateammonium on Z. mays, O. sativa, A. esculentus, and A. gangeticus were assessed visually at $1,7,14$, and 21 DAS (Table 5) to find out the selectivity of the applied herbicide. WeedLock, glyphosate isopropyl-amine, and glufosinate-ammonium all exhibited significant $(p \leq 0.05)$ injury levels on the tested crops when compared to the untreated (control) (Table 5). Crop injury level increased or remained constant over time from 1 to 7 days after spray. The visual injury scales of herbicide-treated crops were significantly greater than untreated (control). At 1 DAS, all tested crops were very heavily damaged with an injury scale rating of 8.00 except for $Z$. mays which had a slightly lower injury (7.75), when WeedLock was applied in $\mathrm{T}_{2}\left(1345.50 \mathrm{~L} \mathrm{ha}^{-1}\right)$. However, at $7 \mathrm{DAS}, O$. sativa, A. esculentus, and $A$. gangeticus were completely killed with an injury rating of 9.00 following application of WeedLock, glyphosate isopropyl-amine, and glufosinate-ammonium at 1345.50, 2, and $2 \mathrm{~L} \mathrm{ha}^{-1}$, respectively. At 21 DAS, the phytotoxicity of WeedLock on tested crops at a lower rate was significantly different from glyphosate isopropyl-amine and glufosinate- 
ammonium. The injury scale rating of Z. mays, O. sativa, A. esculentus, and A. gangeticus was recorded as $3.25,4.75,6.50$, and 4.25 , respectively, at a lower dose of $\mathrm{T}_{1}\left(672.75 \mathrm{~L} \mathrm{ha}^{-1}\right)$ of WeedLock, in comparison to complete inhibition observed in glyphosate isopropyl-amine and glufosinate-ammonium in $\mathrm{T}_{1}\left(1 \mathrm{~L} \mathrm{ha}^{-1}\right)$. On the contrary, at 21 DAS, no significant difference between WeedLock, glyphosate isopropyl-amine, and glufosinate-ammonium was observed at a higher rate $\left(\mathrm{T}_{3}\right)$, as all tested crops were completely killed.

Table 4. Effects of WeedLock, glyphosate isopropyl-amine, and glufosinate-ammonium on weed control efficiency (\%).

\begin{tabular}{|c|c|c|c|c|}
\hline \multirow{2}{*}{ Test Weeds } & \multirow{2}{*}{ Doses } & \multicolumn{3}{|c|}{ Weed Control Efficiency (\%) } \\
\hline & & WeedLock & Glyphosate Isopropyl-Amine & Glufosinate-Ammonium \\
\hline \multirow{4}{*}{ Ageratum conyzoides } & $\mathrm{T}_{0}$ & $0.00 \mathrm{a} \pm 0$ & $0.00 \mathrm{a} \pm 0$ & $0.00 \mathrm{a} \pm 0$ \\
\hline & $\mathrm{T}_{1}$ & $90.66 \mathrm{a} \pm 0.86$ & $90.86 a \pm 0.84$ & $89.09 a \pm 0.93$ \\
\hline & $\mathrm{T}_{2}$ & $97.56 \mathrm{a} \pm 0.38$ & $97.61 \mathrm{a} \pm 0.32$ & $97.19 \mathrm{a} \pm 0.36$ \\
\hline & $\mathrm{T}_{3}$ & $98.49 \mathrm{ab} \pm 0.08$ & $98.55 \mathrm{a} \pm 0.09$ & $98.16 b \pm 0.17$ \\
\hline \multirow{4}{*}{ Euphorbia hirta } & $\mathrm{T}_{0}$ & $0.00 \mathrm{a} \pm 0$ & $0.00 \mathrm{a} \pm 0$ & $0.00 \mathrm{a} \pm 0$ \\
\hline & $\mathrm{T}_{1}$ & $84.82 \mathrm{~b} \pm 0.45$ & $89.88 \mathrm{a} \pm 0.36$ & $89.32 \mathrm{a} \pm 0.56$ \\
\hline & $\mathrm{T}_{2}$ & $89.04 b \pm 0.55$ & $91.30 \mathrm{a} \pm 0.31$ & $90.65 \mathrm{a} \pm 0.34$ \\
\hline & $\mathrm{T}_{3}$ & $92.12 b \pm 0.40$ & $96.99 \mathrm{a} \pm 0.45$ & $96.18 \mathrm{a} \pm 0.55$ \\
\hline \multirow{4}{*}{ Eleusine indica } & $\mathrm{T}_{0}$ & $0.00 \mathrm{a} \pm 0$ & $0.00 \mathrm{a} \pm 0$ & $0.00 \mathrm{a} \pm 0$ \\
\hline & $\mathrm{T}_{1}$ & $90.43 a \pm 0.79$ & $92.12 \mathrm{a} \pm 1.09$ & $91.68 \mathrm{a} \pm 0.50$ \\
\hline & $\mathrm{T}_{2}$ & $97.36 \mathrm{a} \pm 0.32$ & $96.69 \mathrm{ab} \pm 0.18$ & $96.38 b \pm 0.27$ \\
\hline & $\mathrm{T}_{3}$ & $98.29 \mathrm{a} \pm 0.14$ & $98.42 \mathrm{a} \pm 0.12$ & $97.47 \mathrm{~b} \pm 0.34$ \\
\hline \multirow{4}{*}{ Axonopus compressus } & $\mathrm{T}_{0}$ & $0.00 \mathrm{a} \pm 0$ & $0.00 \mathrm{a} \pm 0$ & $0.00 \mathrm{a} \pm 0$ \\
\hline & $\mathrm{T}_{1}$ & $83.39 b \pm 0.64$ & $91.93 \mathrm{a} \pm 0.22$ & $90.57 \mathrm{a} \pm 0.38$ \\
\hline & $\mathrm{T}_{2}$ & $90.92 b \pm 0.36$ & $94.36 \mathrm{a} \pm 0.44$ & $92.16 b \pm 0.61$ \\
\hline & $\mathrm{T}_{3}$ & $97.07 \mathrm{a} \pm 0.39$ & $96.73 \mathrm{ab} \pm 0.29$ & $95.69 \mathrm{~b} \pm 0.48$ \\
\hline \multirow{4}{*}{ Cyperus iria } & $\mathrm{T}_{0}$ & $0.00 \mathrm{a} \pm 0$ & $0.00 \mathrm{a} \pm 0$ & $0.00 \mathrm{a} \pm 0$ \\
\hline & $\mathrm{T}_{1}$ & $85.21 b \pm 1.53$ & $93.71 \mathrm{a} \pm 0.35$ & $87.37 \mathrm{~b} \pm 0.33$ \\
\hline & $\mathrm{T}_{2}$ & $95.08 \mathrm{ab} \pm 0.40$ & $96.42 \mathrm{a} \pm 0.36$ & $92.55 b \pm 1.51$ \\
\hline & $\mathrm{T}_{3}$ & $98.20 \mathrm{a} \pm 0.19$ & $98.35 a \pm 0.23$ & $96.32 \mathrm{~b} \pm 0.44$ \\
\hline \multirow{4}{*}{ Fimbristylis miliacea } & $\mathrm{T}_{0}$ & $0.00 \mathrm{a} \pm 0$ & $0.00 \mathrm{a} \pm 0$ & $0.00 \mathrm{a} \pm 0$ \\
\hline & $\mathrm{T}_{1}$ & $78.70 b \pm 1.62$ & $85.66 \mathrm{a} \pm 1.41$ & $85.41 \mathrm{a} \pm 2.44$ \\
\hline & $\mathrm{T}_{2}$ & $83.62 b \pm 1.32$ & $89.45 a \pm 1.46$ & $89.39 a \pm 1.56$ \\
\hline & $\mathrm{T}_{3}$ & $91.87 \mathrm{a} \pm 1.45$ & $94.34 \mathrm{a} \pm 1.13$ & $92.20 \mathrm{a} \pm 1.31$ \\
\hline
\end{tabular}

Data are expressed as mean \pm standard error. Means having the same letter among the herbicides for each dose in a row are not significantly different at $p<0.05$. Here, DAS: day after spray; $\mathrm{T}_{0}$ : untreated (control), $\mathrm{T}_{1}: 672.75 \mathrm{~L} \mathrm{ha}^{-1}(\mathrm{WL}), 1 \mathrm{~L} \mathrm{ha}^{-1}\left(\mathrm{GLY}\right.$ and GLU), $\mathrm{T}_{2}: 1345.50 \mathrm{~L}$ ha ${ }^{-1}$ (WL), $2 \mathrm{~L} \mathrm{ha}^{-1}$ (GLY and GLU); $\mathrm{T}_{3}$ : $2691.00 \mathrm{~L} \mathrm{ha}^{-1}$ (WL), $4 \mathrm{~L} \mathrm{ha}^{-1}$ (GLY and GLU). WL: WeedLock, GLY: glyphosate isopropyl-amine, GLU: glufosinate-ammonium.

3.1.5. Effects of WeedLock, Glyphosate Isopropyl-Amine, and Glufosinate-Ammonium on Fresh Weight, Dry Weight, and Growth Reduction of Selected Crop Species

Fresh weight, dry weight, and growth reduction of all tested crops were significantly $(p \leq 0.05$ ) decreased by all the applied herbicides (Table 6). The foliar spray of WeedLock at a lower/half rate $\mathrm{T}_{1}\left(672.75 \mathrm{~L} \mathrm{ha}^{-1}\right)$ reduced the fresh weight $62.37 \%$ in 0. sativa, $59.19 \%$ in Z. mays, $92.62 \%$ in A. esculentus, and $54.50 \%$ in A. gangeticus. Among the crops, a noticeable fresh weight reduction was evident in A. esculentus in $\mathrm{T}_{2}\left(1345.50 \mathrm{~L} \mathrm{ha}^{-1}\right)$ of WeedLock with a reduction index of $96.56 \%$, followed by $94.20 \%, 92.58 \%$, and $85.10 \%$ in Z . mays, A. gangeticus, and O. sativa, respectively. WeedLock showed a similar effect on the fresh weight reduction of tested crops compared to glyphosate isopropyl-amine and glufosinateammonium at the highest rate $\left(\mathrm{T}_{3}\right)$. At a $2691.00 \mathrm{~L} \mathrm{ha}^{-1}\left(\mathrm{~T}_{3}\right)$ application rate, WeedLock caused $98.54 \%, 95.36 \%, 98.22 \%$, and $95.27 \%$ reductions in fresh weight of Z. mays, O. sativa, A. esculentus, and A. gangeticus, respectively. On the other hand, $97.42 \%$ to $98.75 \%$, and $96.95 \%$ to $98.87 \%$ fresh weight reductions for all tested crops were achieved by glyphosate isopropyl-amine and glufosinate-ammonium at $4 \mathrm{~L} \mathrm{ha}^{-1}\left(\mathrm{~T}_{3}\right)$, respectively. 
Table 5. Effects of WeedLock, glyphosate isopropyl-amine, and glufosinate-ammonium on an injury scale of tested crop species.

\begin{tabular}{|c|c|c|c|c|c|c|c|c|c|c|c|c|c|}
\hline \multirow{3}{*}{ Test Crops } & \multirow{3}{*}{ Doses } & \multicolumn{12}{|c|}{ Visual Injury (Scale) } \\
\hline & & \multicolumn{3}{|c|}{1 DAS } & \multicolumn{3}{|c|}{7 DAS } & \multicolumn{3}{|c|}{14 DAS } & \multicolumn{3}{|c|}{21 DAS } \\
\hline & & WL & GLY & GLU & WL & GLY & GLU & WL & GLY & GLU & WL & GLY & GLU \\
\hline Zea mays & $\begin{array}{l}\mathrm{T}_{0} \\
\mathrm{~T}_{1} \\
\mathrm{~T}_{2} \\
\mathrm{~T}_{3}\end{array}$ & $\begin{array}{c}1.00 \mathrm{a} \pm 0 \\
3.75 \mathrm{a} \pm 0.48 \\
7.75 \mathrm{a} \pm 0.25 \\
8.00 \mathrm{a} \pm 0\end{array}$ & $\begin{array}{l}1.00 \mathrm{a} \pm 0 \\
2.00 \mathrm{~b} \pm 0 \\
2.00 \mathrm{c} \pm 0 \\
2.00 \mathrm{c} \pm 0\end{array}$ & $\begin{array}{c}1.00 \mathrm{a} \pm 0 \\
2.00 \mathrm{~b} \pm 0 \\
2.75 \mathrm{~b} \pm 0.25 \\
2.75 \mathrm{~b} \pm 0.25\end{array}$ & $\begin{array}{c}1.00 \mathrm{a} \pm 0 \\
3.25 \mathrm{~b} \pm 0.25 \\
8.75 \mathrm{a} \pm 0.25 \\
9.00 \mathrm{a} \pm 0\end{array}$ & $\begin{array}{l}1.00 \mathrm{a} \pm 0 \\
9.00 \mathrm{a} \pm 0 \\
9.00 \mathrm{a} \pm 0 \\
9.00 \mathrm{a} \pm 0\end{array}$ & $\begin{array}{l}1.00 \mathrm{a} \pm 0 \\
9.00 \mathrm{a} \pm 0 \\
9.00 \mathrm{a} \pm 0 \\
9.00 \mathrm{a} \pm 0\end{array}$ & $\begin{array}{c}1.00 \mathrm{a} \pm 0 \\
3.25 \mathrm{~b} \pm 0.25 \\
8.75 \mathrm{a} \pm 0.25 \\
9.00 \mathrm{a} \pm 0\end{array}$ & $\begin{array}{l}1.00 \mathrm{a} \pm 0 \\
9.00 \mathrm{a} \pm 0 \\
9.00 \mathrm{a} \pm 0 \\
9.00 \mathrm{a} \pm 0\end{array}$ & $\begin{array}{l}1.00 \mathrm{a} \pm 0 \\
9.00 \mathrm{a} \pm 0 \\
9.00 \mathrm{a} \pm 0 \\
9.00 \mathrm{a} \pm 0\end{array}$ & $\begin{array}{c}1.00 \mathrm{a} \pm 0 \\
3.25 \mathrm{~b} \pm 0.25 \\
8.75 \mathrm{a} \pm 0.25 \\
9.00 \mathrm{a} \pm 0\end{array}$ & $\begin{array}{l}1.00 \mathrm{a} \pm 0 \\
9.00 \mathrm{a} \pm 0 \\
9.00 \mathrm{a} \pm 0 \\
9.00 \mathrm{a} \pm 0\end{array}$ & $\begin{array}{c}1.00 \mathrm{a} \pm 0 \\
9.00 \pm 0 \\
9.00 \mathrm{a} \pm 0 \\
9.00 \mathrm{a} \pm\end{array}$ \\
\hline Oryza sativa & $\begin{array}{l}\mathrm{T}_{0} \\
\mathrm{~T}_{1} \\
\mathrm{~T}_{2} \\
\mathrm{~T}_{3} \\
\end{array}$ & $\begin{array}{c}1.00 \mathrm{a} \pm 0 \\
5.75 \mathrm{a} \pm 0.48 \\
8.00 \mathrm{a} \pm 0 \\
8.00 \mathrm{a} \pm 0\end{array}$ & $\begin{array}{l}1.00 \mathrm{a} \pm 0 \\
2.00 \mathrm{~b} \pm 0 \\
2.00 \mathrm{~b} \pm 0 \\
2.00 \mathrm{~b} \pm 0\end{array}$ & $\begin{array}{c}1.00 \mathrm{a} \pm 0 \\
2.00 \mathrm{~b} \pm 0 \\
2.50 \mathrm{~b} \pm 0.29 \\
2.50 \mathrm{~b} \pm 0.29\end{array}$ & $\begin{array}{c}1.00 \mathrm{a} \pm 0 \\
5.75 \mathrm{~b} \pm 0.48 \\
9.00 \mathrm{a} \pm 0 \\
9.00 \mathrm{a} \pm 0\end{array}$ & $\begin{array}{l}1.00 \mathrm{a} \pm 0 \\
9.00 \mathrm{a} \pm 0 \\
9.00 \mathrm{a} \pm 0 \\
9.00 \mathrm{a} \pm 0\end{array}$ & $\begin{array}{l}1.00 \mathrm{a} \pm 0 \\
9.00 \mathrm{a} \pm 0 \\
9.00 \mathrm{a} \pm 0 \\
9.00 \mathrm{a} \pm 0\end{array}$ & $\begin{array}{c}1.00 \mathrm{a} \pm 0 \\
4.75 \mathrm{~b} \pm 0.48 \\
9.00 \mathrm{a} \pm 0 \\
9.00 \mathrm{a} \pm 0\end{array}$ & $\begin{array}{l}1.00 \mathrm{a} \pm 0 \\
9.00 \mathrm{a} \pm 0 \\
9.00 \mathrm{a} \pm 0 \\
9.00 \mathrm{a} \pm 0\end{array}$ & $\begin{array}{l}1.00 \mathrm{a} \pm 0 \\
9.00 \mathrm{a} \pm 0 \\
9.00 \mathrm{a} \pm 0 \\
9.00 \mathrm{a} \pm 0\end{array}$ & $\begin{array}{c}1.00 \mathrm{a} \pm 0 \\
4.75 \mathrm{~b} \pm 0.48 \\
9.00 \mathrm{a} \pm 0 \\
9.00 \mathrm{a} \pm 0\end{array}$ & $\begin{array}{l}1.00 \mathrm{a} \pm 0 \\
9.00 \mathrm{a} \pm 0 \\
9.00 \mathrm{a} \pm 0 \\
9.00 \mathrm{a} \pm 0\end{array}$ & $\begin{array}{l}1.00 \mathrm{a} \pm 0 \\
9.00 \mathrm{a} \pm 0 \\
9.00 \mathrm{a} \pm \\
9.00 \mathrm{a} \pm\end{array}$ \\
\hline $\begin{array}{l}\text { Abelmoschus } \\
\text { esculentus }\end{array}$ & $\begin{array}{l}\mathrm{T}_{0} \\
\mathrm{~T}_{1} \\
\mathrm{~T}_{2} \\
\mathrm{~T}_{3} \\
\end{array}$ & $\begin{array}{c}1.00 \mathrm{a} \pm 0 \\
7.50 \mathrm{a} \pm 0.29 \\
8.00 \mathrm{a} \pm 0 \\
8.00 \mathrm{a} \pm 0\end{array}$ & $\begin{array}{l}1.00 \mathrm{a} \pm 0 \\
2.00 \mathrm{~b} \pm 0 \\
2.00 \mathrm{~b} \pm 0 \\
2.00 \mathrm{c} \pm 0\end{array}$ & $\begin{array}{c}1.00 \mathrm{a} \pm 0 \\
2.00 \mathrm{~b} \pm 0 \\
2.50 \mathrm{~b} \pm 0.29 \\
2.75 \mathrm{~b} \pm 0.25\end{array}$ & $\begin{array}{c}1.00 \mathrm{a} \pm 0 \\
7.25 \mathrm{~b} \pm 0.48 \\
9.00 \mathrm{a} \pm 0 \\
9.00 \mathrm{a} \pm 0\end{array}$ & $\begin{array}{l}1.00 \mathrm{a} \pm 0 \\
9.00 \mathrm{a} \pm 0 \\
9.00 \mathrm{a} \pm 0 \\
9.00 \mathrm{a} \pm 0\end{array}$ & $\begin{array}{l}1.00 \mathrm{a} \pm 0 \\
9.00 \mathrm{a} \pm 0 \\
9.00 \mathrm{a} \pm 0 \\
9.00 \mathrm{a} \pm 0\end{array}$ & $\begin{array}{c}1.00 \mathrm{a} \pm 0 \\
6.50 \mathrm{~b} \pm 0.29 \\
9.00 \mathrm{a} \pm 0 \\
9.00 \mathrm{a} \pm 0\end{array}$ & $\begin{array}{l}1.00 \mathrm{a} \pm 0 \\
9.00 \mathrm{a} \pm 0 \\
9.00 \mathrm{a} \pm 0 \\
9.00 \mathrm{a} \pm 0\end{array}$ & $\begin{array}{l}1.00 \mathrm{a} \pm 0 \\
9.00 \mathrm{a} \pm 0 \\
9.00 \mathrm{a} \pm 0 \\
9.00 \mathrm{a} \pm 0\end{array}$ & $\begin{array}{c}1.00 \mathrm{a} \pm 0 \\
6.50 \mathrm{~b} \pm 0.29 \\
9.00 \mathrm{a} \pm 0 \\
9.00 \mathrm{a} \pm 0\end{array}$ & $\begin{array}{l}1.00 \mathrm{a} \pm 0 \\
9.00 \mathrm{a} \pm 0 \\
9.00 \mathrm{a} \pm 0 \\
9.00 \mathrm{a} \pm 0\end{array}$ & $\begin{array}{l}1.00 \mathrm{a} \pm 0 \\
9.00 \mathrm{a} \pm \\
9.00 \mathrm{a} \pm \\
9.00 \mathrm{a} \pm\end{array}$ \\
\hline $\begin{array}{l}\text { Amaranthus } \\
\text { gangeticus }\end{array}$ & $\begin{array}{l}\mathrm{T}_{0} \\
\mathrm{~T}_{1} \\
\mathrm{~T}_{2} \\
\mathrm{~T}_{3}\end{array}$ & $\begin{array}{c}1.00 \mathrm{a} \pm 0 \\
5.00 \mathrm{a} \pm 0.41 \\
8.00 \mathrm{a} \pm 0 \\
8.00 \mathrm{a} \pm 0\end{array}$ & $\begin{array}{l}1.00 \mathrm{a} \pm 0 \\
2.00 \mathrm{~b} \pm 0 \\
2.00 \mathrm{~b} \pm 0 \\
2.00 \mathrm{c} \pm 0\end{array}$ & $\begin{array}{c}1.00 \mathrm{a} \pm 0 \\
2.00 \mathrm{~b} \pm 0 \\
2.50 \mathrm{~b} \pm 0.29 \\
2.75 \mathrm{~b} \pm 0.25\end{array}$ & $\begin{array}{c}1.00 \mathrm{a} \pm 0 \\
4.50 \mathrm{~b} \pm 0.29 \\
9.00 \mathrm{a} \pm 0 \\
9.00 \mathrm{a} \pm 0\end{array}$ & $\begin{array}{l}1.00 \mathrm{a} \pm 0 \\
9.00 \mathrm{a} \pm 0 \\
9.00 \mathrm{a} \pm 0 \\
9.00 \mathrm{a} \pm 0\end{array}$ & $\begin{array}{l}1.00 \mathrm{a} \pm 0 \\
9.00 \mathrm{a} \pm 0 \\
9.00 \mathrm{a} \pm 0 \\
9.00 \mathrm{a} \pm 0\end{array}$ & $\begin{array}{c}1.00 \mathrm{a} \pm 0 \\
4.25 \mathrm{~b} \pm 0.25 \\
9.00 \mathrm{a} \pm 0 \\
9.00 \mathrm{a} \pm 0\end{array}$ & $\begin{array}{l}1.00 \mathrm{a} \pm 0 \\
9.00 \mathrm{a} \pm 0 \\
9.00 \mathrm{a} \pm 0 \\
9.00 \mathrm{a} \pm 0\end{array}$ & $\begin{array}{l}1.00 \mathrm{a} \pm 0 \\
9.00 \mathrm{a} \pm 0 \\
9.00 \mathrm{a} \pm 0 \\
9.00 \mathrm{a} \pm 0\end{array}$ & $\begin{array}{c}1.00 \mathrm{a} \pm 0 \\
4.25 \mathrm{~b} \pm 0.25 \\
9.00 \mathrm{a} \pm 0 \\
9.00 \mathrm{a} \pm 0\end{array}$ & $\begin{array}{l}1.00 \mathrm{a} \pm 0 \\
9.00 \mathrm{a} \pm 0 \\
9.00 \mathrm{a} \pm 0 \\
9.00 \mathrm{a} \pm 0\end{array}$ & $\begin{array}{l}1.00 \mathrm{a} \pm 0 \\
9.00 \mathrm{a} \pm \\
9.00 \mathrm{a} \pm \\
9.00 \mathrm{a} \pm\end{array}$ \\
\hline
\end{tabular}

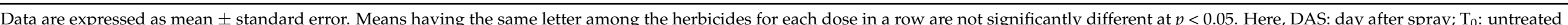

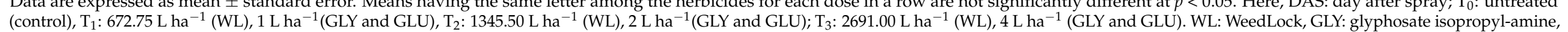

GLU: glufosinate-ammonium. 


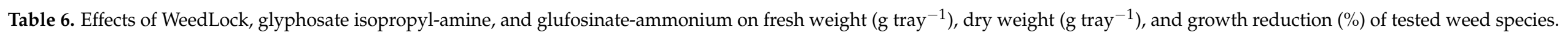

\begin{tabular}{|c|c|c|c|c|c|c|c|c|c|c|}
\hline \multirow{2}{*}{ Test Crops } & \multirow{2}{*}{ Doses } & \multicolumn{3}{|c|}{ Fresh Weight (g) } & \multicolumn{3}{|c|}{ Dry Weight (g) } & \multicolumn{3}{|c|}{ Growth Reduction (\%) } \\
\hline & & WL & GLY & GLU & WL & GLY & GLU & WL & GLY & GLU \\
\hline Zea mays & $\begin{array}{l}\mathrm{T}_{0} \\
\mathrm{~T}_{1} \\
\mathrm{~T}_{2} \\
\mathrm{~T}_{3}\end{array}$ & $\begin{array}{c}542.89 \mathrm{~b} \pm 3.47 \\
221.52 \mathrm{a} \pm 1.89 \\
31.47 \mathrm{a} \pm 1.23 \\
7.93 \mathrm{~b} \pm 0.39\end{array}$ & $\begin{array}{c}549.34 \mathrm{ab} \pm 3.18 \\
23.12 \mathrm{~b} \pm 0.64 \\
14.13 \mathrm{~b} \pm 0.17 \\
8.62 \mathrm{~b} \pm 0.20\end{array}$ & $\begin{array}{c}556.72 \mathrm{a} \pm 3.82 \\
20.28 \mathrm{~b} \pm 0.61 \\
15.26 \mathrm{~b} \pm 0.55 \\
10.96 \mathrm{a} \pm 0.66\end{array}$ & $\begin{array}{c}93.58 \mathrm{~b} \pm 3.52 \\
62.89 \mathrm{a} \pm 2.13 \\
3.17 \mathrm{a} \pm 1.16 \\
0.94 \mathrm{a} \pm 0.24\end{array}$ & $\begin{array}{c}100.86 \mathrm{ab} \pm 2.02 \\
2.45 \mathrm{~b} \pm 0.36 \\
1.95 \mathrm{a} \pm 0.11 \\
1.00 \mathrm{a} \pm 0.24\end{array}$ & $\begin{array}{c}103.08 \mathrm{a} \pm 1.44 \\
2.27 \mathrm{~b} \pm 0.22 \\
2.09 \mathrm{a} \pm 0.22 \\
1.36 \mathrm{a} \pm 0.29\end{array}$ & $\begin{array}{c}0.00 \mathrm{a} \pm 0 \\
32.26 \mathrm{~b} \pm 4.81 \\
96.64 \mathrm{a} \pm 1.16 \\
98.99 \mathrm{a} \pm 0.27\end{array}$ & $\begin{array}{c}0.00 \mathrm{a} \pm 0 \\
97.55 \mathrm{a} \pm 0.40 \\
98.06 \mathrm{a} \pm 0.12 \\
99.00 \mathrm{a} \pm 0.25\end{array}$ & $\begin{array}{c}0.00 \mathrm{a} \pm 0 \\
97.80 \mathrm{a} \pm 0.19 \\
97.98 \mathrm{a} \pm 0.19 \\
98.69 \mathrm{a} \pm 0.26\end{array}$ \\
\hline Oryza sativa & $\begin{array}{l}\mathrm{T}_{0} \\
\mathrm{~T}_{1} \\
\mathrm{~T}_{2} \\
\mathrm{~T}_{3}\end{array}$ & $\begin{array}{c}45.13 a \pm 0.49 \\
16.96 a \pm 0.47 \\
6.71 a \pm 0.33 \\
2.09 a \pm 0.11\end{array}$ & $\begin{array}{l}43.56 \mathrm{a} \pm 0.62 \\
4.20 \mathrm{~b} \pm 0.25 \\
2.12 \mathrm{~b} \pm 0.19 \\
1.12 \mathrm{~b} \pm 0.10 \\
\end{array}$ & $\begin{array}{c}44.74 \mathrm{a} \pm 0.35 \\
4.32 \mathrm{~b} \pm 0.22 \\
2.04 \mathrm{~b} \pm 0.12 \\
1.36 \mathrm{~b} \pm 0.06\end{array}$ & $\begin{array}{c}11.22 \mathrm{a} \pm 0.13 \\
3.71 \mathrm{a} \pm 0.10 \\
1.65 \mathrm{a} \pm 0.09 \\
0.83 \mathrm{a} \pm 0.07\end{array}$ & $\begin{array}{c}10.93 \mathrm{a} \pm 0 \\
1.22 \mathrm{~b} \pm 0.21 \\
0.79 \mathrm{~b} \pm 0.08 \\
0.41 \mathrm{~b} \pm 0.04\end{array}$ & $\begin{array}{c}11.16 \mathrm{a} \pm 0.20 \\
1.29 \mathrm{~b} \pm 0.08 \\
0.84 \mathrm{~b} \pm 0.04 \\
0.50 \mathrm{~b} \pm 0.05\end{array}$ & $\begin{array}{c}0.00 \mathrm{a} \pm 0 \\
66.88 \mathrm{~b} \pm 1.03 \\
85.31 \mathrm{~b} \pm 0.86 \\
92.59 \mathrm{~b} \pm 0.53\end{array}$ & $\begin{array}{c}0.00 \mathrm{a} \pm 0 \\
88.85 \mathrm{a} \pm 0.58 \\
92.71 \mathrm{a} \pm 0.71 \\
96.23 \mathrm{a} \pm 0.44\end{array}$ & $\begin{array}{c}0.00 \mathrm{a} \pm 0 \\
88.38 \mathrm{a} \pm 0.79 \\
92.50 \mathrm{a} \pm 0.26 \\
95.48 \mathrm{a} \pm 0.37\end{array}$ \\
\hline Abelmoschus esculentus & $\begin{array}{l}\mathrm{T}_{0} \\
\mathrm{~T}_{1} \\
\mathrm{~T}_{2} \\
\mathrm{~T}_{3}\end{array}$ & $\begin{array}{c}355.68 \mathrm{a} \pm 3.47 \\
26.24 \mathrm{a} \pm 0.50 \\
12.22 \mathrm{a} \pm 0.56 \\
6.33 \mathrm{a} \pm 0.28\end{array}$ & $\begin{array}{c}342.53 b \pm 3.90 \\
10.34 c \pm 0.35 \\
6.90 b \pm 0.30 \\
4.35 b \pm 0.17\end{array}$ & $\begin{array}{c}341.83 b \pm 3.39 \\
12.59 b \pm 0.25 \\
7.22 b \pm 0.21 \\
3.85 b \pm 0.13\end{array}$ & $\begin{array}{c}70.49 a \pm 1.66 \\
2.95 a \pm 0.28 \\
1.76 a \pm 0.15 \\
0.77 a \pm 0.09\end{array}$ & $\begin{array}{c}68.27 \mathrm{a} \pm 3.36 \\
1.94 \mathrm{~b} \pm 0.11 \\
1.15 \mathrm{~b} \pm 0.08 \\
0.79 \mathrm{a} \pm 0.02 \\
\end{array}$ & $\begin{array}{c}66.95 a \pm 2.85 \\
2.02 b \pm 0.07 \\
1.18 b \pm 0.09 \\
0.73 a \pm 0.07\end{array}$ & $\begin{array}{c}0.00 \mathrm{a} \pm 0 \\
95.84 \mathrm{~b} \pm 0.31 \\
97.51 \mathrm{~b} \pm 0.17 \\
98.90 \mathrm{a} \pm 0.10\end{array}$ & $\begin{array}{c}0.00 \mathrm{a} \pm 0 \\
97.16 \mathrm{a} \pm 0.05 \\
98.32 \mathrm{a} \pm 0.05 \\
98.83 \mathrm{a} \pm 0.05\end{array}$ & $\begin{array}{c}0.00 \mathrm{a} \pm 0 \\
96.98 \mathrm{a} \pm 0.06 \\
98.24 \mathrm{a} \pm 0.08 \\
98.92 \mathrm{a} \pm 0.07 \\
\end{array}$ \\
\hline Amaranthus gangeticus & $\begin{array}{l}\mathrm{T}_{0} \\
\mathrm{~T}_{1} \\
\mathrm{~T}_{2} \\
\mathrm{~T}_{3}\end{array}$ & $\begin{array}{c}86.02 \mathrm{a} \pm 1.00 \\
39.14 \mathrm{a} \pm 0.64 \\
6.39 \mathrm{a} \pm 0.23 \\
4.07 \mathrm{a} \pm 0.10\end{array}$ & $\begin{array}{l}84.42 \mathrm{a} \pm 0.97 \\
5.29 \mathrm{~b} \pm 0.20 \\
3.38 \mathrm{c} \pm 0.21 \\
1.09 \mathrm{c} \pm 0.10\end{array}$ & $\begin{array}{c}85.09 \mathrm{a} \pm 1.05 \\
6.67 \mathrm{~b} \pm 0.40 \\
4.52 \mathrm{~b} \pm 0.23 \\
1.66 \mathrm{~b} \pm 0.12\end{array}$ & $\begin{array}{c}21.10 a \pm 0.68 \\
10.23 a \pm 0.52 \\
1.39 a \pm 0.05 \\
0.95 a \pm 0.04\end{array}$ & $\begin{array}{c}20.42 \mathrm{a} \pm 0.48 \\
1.79 \mathrm{~b} \pm 0.06 \\
1.36 \mathrm{a} \pm 0.04 \\
0.92 \mathrm{a} \pm 0.04\end{array}$ & $\begin{array}{c}20.49 \mathrm{a} \pm 0.27 \\
1.82 \mathrm{~b} \pm 0.07 \\
1.39 \mathrm{a} \pm 0.03 \\
0.96 \mathrm{a} \pm 0.04\end{array}$ & $\begin{array}{c}0.00 \mathrm{a} \pm 0 \\
51.42 \mathrm{~b} \pm 2.84 \\
93.38 \mathrm{a} \pm 0.27 \\
95.49 \mathrm{a} \pm 0.24\end{array}$ & $\begin{array}{c}0.00 \mathrm{a} \pm 0 \\
91.20 \mathrm{a} \pm 0.41 \\
93.31 \mathrm{a} \pm 0.28 \\
95.46 \mathrm{a} \pm 0.21\end{array}$ & $\begin{array}{c}0.00 \mathrm{a} \pm 0 \\
91.09 \mathrm{a} \pm 0.43 \\
93.20 \mathrm{a} \pm 0.20 \\
95.33 \mathrm{a} \pm 0.19\end{array}$ \\
\hline
\end{tabular}

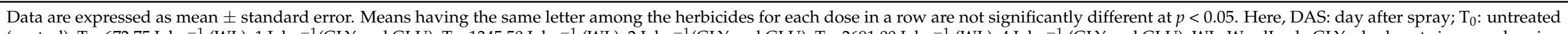

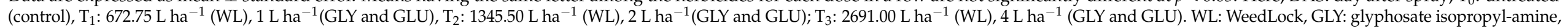

GLU: glufosinate-ammonium. 
The dry weight was measured at 21 days after spray and significantly $(p \leq 0.05)$ differed applying WeedLock, glyphosate isopropyl-amine, and glufosinate-ammonium (Table 6). A $672.75 \mathrm{~L} \mathrm{ha}^{-1}\left(\mathrm{~T}_{1}\right)$ application rate of WeedLock caused $66.88 \%, 32.26 \%$, $95.83 \%$, and $51.42 \%$ reduction in the dry weight of O. sativa, Z. mays, A. esculentus, and $A$. gangeticus, respectively. At the application rate of $\mathrm{T}_{2}\left(1345.50 \mathrm{~L} \mathrm{ha}^{-1}\right)$, WeedLock produced a significant growth inhibition in $O$. sativa and $A$. esculentus with a reduction of $85.31 \%$ and $97.51 \%$, but no significant difference was recorded for Z. mays and A. gangeticus compared to glyphosate isopropyl-amine and glufosinate-ammonium at $\mathrm{T}_{2}\left(2 \mathrm{~L} \mathrm{ha}^{-1}\right)$. The lowest growth inhibition in $\mathrm{T}_{1}$ was recorded in $Z$. mays, where WeedLock $\left(672.75 \mathrm{~L} \mathrm{ha}^{-1}\right)$ yielded $32.26 \%$ reduction while glyphosate isopropyl-amine $\left(1 \mathrm{~L} \mathrm{ha}^{-1}\right)$ and glufosinate-ammonium $\left(1 \mathrm{~L} \mathrm{ha}^{-1}\right.$ ) caused $97.55 \%$ and $97.80 \%$, respectively. Thus, dry weights of all crops were reduced by more than $85.29 \%$ in $\mathrm{T}_{2}\left(1345.50 \mathrm{~L} \mathrm{ha}^{-1}\right)$ of WeedLock compared to untreated (control). At a higher dose $\left(\mathrm{T}_{3}\right)$, WeedLock $\left(2691.00 \mathrm{~L} \mathrm{ha}^{-1}\right)$, glyphosate isopropyl-amine $\left(4 \mathrm{~L} \mathrm{ha}^{-1}\right)$, and glufosinate-ammonium $\left(4 \mathrm{~L} \mathrm{ha}^{-1}\right)$ reduced the growth of all tested crops by $92.59-98.99 \%, 95.46-99.00 \%$, and $95.33-98.92 \%$, respectively. This shows that WeedLock gave a similar growth reduction compared to glyphosate isopropyl-amine and glufosinateammonium for all crop species.

\subsection{Field Trial Experiment}

\subsubsection{Floristic Weed Composition}

In the current study, the experimental plots were populated with 32 weed species indicating a mixture of broadleaf weeds, grasses, and sedges. The weed composition was dominated by 13 broadleaf species comprising 8 different families, 13 types of grasses, and only 6 sedges species (Table 7). The summed dominance ratio (SDR) value of Eleutheranthera ruderalis (Sw.) Sch.Bip. was the highest and recorded as the most predominant species in all herbicide treatments. In $\mathrm{T}_{0}$ (untreated), E. ruderalis was the most dominant weed with an SDR value of $16.86 \%$, followed by $A$. conyzoides $(14.91 \%)$ and F. miliacea $(14.90 \%)$, while Mimosa pudica L. (1.80\%) was listed as the least dominant species. Phyllanthus amarus Schumach. \& Thonn. was also dominant with a $16.75 \%$ SDR value, and Mimosa invisa Colla was least dominant with a $2.64 \%$ SDR value in $\mathrm{T}_{1}\left(1345.50 \mathrm{~L}^{-1}\right.$ heedLock). Among the grasses, Paspalum conjugatum P.J.Bergius was dominant in $\mathrm{T}_{4}$ plots ( $4 \mathrm{~L} \mathrm{ha}^{-1}$ glyphosate isopropyl-amine) with a high SDR value of $14.57 \%$, followed by Panicum maximum Jacq. $(12.84 \%)$, and Brachiaria mutica (Forssk.) Stapf. (11.03\%). Based on SDR value, sedge weed species Cyperus digitatus Roxb (18.07\%) was the most predominant species in $\mathrm{T}_{5}\left(2 \mathrm{~L} \mathrm{ha}^{-1}\right.$ glufosinate-ammonium), followed by broadleaf weed Desmodium triflorum (L.) DC. (14.25\%) and grass weed species B. mutica $(12.78 \%)$. Therefore, the experimental plots specified a combination of diversified weed species of broadleaves, grasses, and sedges, with the broadleaves being more dominant over grasses and sedges.

\subsubsection{Coefficient of Similarity}

The similarity coefficient between different herbicide treatment plots indicates (Table 8) the species similarity in weed communities. Sorenson's index of similarity values between $38.94 \%$ to $67.79 \%$ indicate a moderate similarity in weed species among all herbicide treatments. A total of $67.79 \%, 66.29 \%$, and $61.19 \%$ similarities were observed between $\mathrm{T}_{0}$ (untreated) with $\mathrm{T}_{5}\left(2 \mathrm{~L} \mathrm{ha}^{-1}\right.$ glufosinate-ammonium), $\mathrm{T}_{2}\left(2691.00 \mathrm{~L} \mathrm{ha}^{-1}\right.$ WeedLock) with $\mathrm{T}_{5}$ (2 L ha ${ }^{-1}$ glufosinate-ammonium), and $\mathrm{T}_{2}$ (2691.00 $\mathrm{L} \mathrm{ha}^{-1}$ WeedLock) with $\mathrm{T}_{3}\left(2 \mathrm{~L} \mathrm{ha}^{-1}\right.$ glyphosate isopropyl-amine), respectively, indicating a close similarity in weed species among $\mathrm{T}_{0}, \mathrm{~T}_{2}, \mathrm{~T}_{3}$, and $\mathrm{T}_{5}$. Meanwhile, the coefficient similarity between $\mathrm{T}_{1}\left(1345.50 \mathrm{~L} \mathrm{ha}^{-1}\right.$ WeedLock) and $\mathrm{T}_{6}\left(4 \mathrm{~L} \mathrm{ha}^{-1}\right.$ glufosinate-ammonium) recorded the lowest value of $38.94 \%$, showing that there was some variability in weed species infesting these plots. 
Table 7. Floristic Weed Composition in the experimental plot with their on summed dominance ratio (\%).

\begin{tabular}{|c|c|c|c|c|c|c|c|c|}
\hline \multirow{2}{*}{$\begin{array}{c}\text { Scientific Name } \\
\text { Broadleaves }\end{array}$} & \multirow[t]{2}{*}{ Family Name } & \multicolumn{7}{|c|}{ Summed Dominance Ratio (\%) } \\
\hline & & $\mathrm{T}_{0}$ & $T_{1}$ & $T_{2}$ & $T_{3}$ & $T_{4}$ & $T_{5}$ & $T_{6}$ \\
\hline Ageratum conyzoides & Asteraceae & 14.91 & 14.22 & 9.02 & 12.91 & 10.06 & 6.97 & 12.63 \\
\hline Ageratum houstonianum Mill. & Asteraceae & 4.53 & 14.35 & 12.25 & 1.69 & 5.05 & 5.58 & - \\
\hline Cleome rutidosperma DC. & Cleomaceae & 10.41 & 16.00 & 13.13 & 14.15 & 18.72 & 1.77 & 12.05 \\
\hline Desmodium triflorum & Fabaceae & - & - & 12.08 & - & 14.34 & 14.25 & 17.36 \\
\hline Eleutheranthera ruderalis & Asteraceae & 16.86 & 12.24 & 12.24 & 12.20 & 17.04 & 10.17 & 9.19 \\
\hline Euphorbia hirta & Euphorbiaceae & 6.81 & 8.27 & 7.68 & 9.58 & 8.99 & 5.01 & 6.23 \\
\hline Hedyotis corymbosa (L.) Lam. & Rubiaceae & 3.35 & 7.36 & 4.72 & - & - & 4.11 & 20.31 \\
\hline Ipomoea aquatica Forssk. & Convolvulaceae & 2.04 & 5.77 & 2.28 & 4.45 & 9.22 & 3.16 & - \\
\hline Ipomoea triloba L. & Convolvulaceae & 4.10 & 7.07 & 7.55 & 6.13 & 5.00 & 10.32 & 10.24 \\
\hline Melochia corchorifolia L. & Malvaceae & 7.60 & 7.92 & 6.75 & 3.68 & 9.44 & 4.31 & - \\
\hline Mimosa invisa & Fabaceae & 8.04 & 2.64 & 4.41 & - & 7.32 & - & 7.07 \\
\hline Mimosa pudica & Fabaceae & 1.80 & 5.73 & 5.53 & - & 4.50 & 6.58 & 4.87 \\
\hline $\begin{array}{l}\text { Phyllanthus amarus } \\
\text { Grasses }\end{array}$ & Phyllanthaceae & 7.18 & 16.75 & 13.34 & 10.33 & 11.00 & 11.50 & 8.32 \\
\hline Brachiaria mutica & Poaceae & 11.57 & 6.20 & 14.78 & - & 11.03 & 12.78 & - \\
\hline Cynodon dactylon (L.) Pers. & Poaceae & 6.23 & 14.62 & 7.51 & 8.27 & 6.41 & 9.60 & 10.85 \\
\hline Digitaria ciliaris (Retz.) Koeler & Poaceae & 6.97 & 9.24 & 8.19 & 8.03 & 6.87 & 8.50 & 9.69 \\
\hline Digitaria fuscescens (J.Presl) Henrard & Poaceae & 9.23 & - & 11.21 & - & - & 9.68 & - \\
\hline Digitaria longiflora (Retz.) Pers. & Poaceae & 12.49 & 8.29 & 5.54 & 8.08 & - & 4.88 & 5.84 \\
\hline Echinochloa colona (L.) Link & Poaceae & 9.21 & - & 6.02 & 8.49 & 6.81 & 7.89 & 7.76 \\
\hline Eleusine indica & Poaceae & - & - & 6.02 & 6.05 & 7.39 & 1.52 & - \\
\hline Ottochloa nodosa (Kunth) Dandy & Poaceae & 4.51 & 14.34 & - & - & 8.04 & 7.81 & 10.54 \\
\hline Panicum maximum & Poaceae & 9.92 & 9.81 & 6.84 & 3.83 & 12.84 & 8.26 & - \\
\hline Paspalum conjugatum & Poaceae & 8.90 & - & 7.73 & - & 14.57 & 4.74 & 11.43 \\
\hline Paspalum distichum L. & Poaceae & 11.69 & - & - & 6.68 & - & 12.76 & 8.70 \\
\hline Paspalum scrobiculatum L. & Poaceae & - & - & 8.47 & 9.92 & 9.67 & 6.77 & 7.39 \\
\hline $\begin{array}{c}\text { Sporobolus diander (Retz.) P.Beauv. } \\
\text { Sedges }\end{array}$ & Poaceae & 4.65 & 6.63 & 4.90 & 6.05 & 9.86 & 9.67 & 9.86 \\
\hline Cyperus digitatus & Cyperaceae & 5.26 & - & 10.10 & 6.68 & 6.44 & 18.07 & 9.02 \\
\hline Cyperus esculentus L. & Cyperaceae & - & 4.07 & 13.95 & - & 12.01 & - & 8.13 \\
\hline Cyperus iria & Cyperaceae & 9.16 & 11.22 & 6.91 & 7.69 & 6.25 & 9.75 & 10.94 \\
\hline Cyperus rotundus L. & Cyperaceae & 9.69 & - & 5.94 & 9.91 & 10.56 & 12.88 & 6.96 \\
\hline Fimbristylis miliacea & Cyperaceae & 14.90 & 12.90 & - & - & 9.57 & 8.13 & 7.62 \\
\hline Rhynchospora corymbosa (L.) Britton & Cyperaceae & 7.38 & 4.06 & - & - & 7.42 & 15.99 & 9.47 \\
\hline
\end{tabular}

Here, $\mathrm{T}_{0}$ : untreated (control), $\mathrm{T}_{1}: 1345.50 \mathrm{~L} \mathrm{ha}^{-1}(\mathrm{WL}), \mathrm{T}_{2}: 2691.00 \mathrm{~L} \mathrm{ha}^{-1}(\mathrm{WL}), \mathrm{T}_{3}: 2 \mathrm{~L} \mathrm{ha}^{-1}(\mathrm{GLY}), \mathrm{T}_{4}: 4 \mathrm{~L} \mathrm{ha}^{-1}(\mathrm{GLY}), \mathrm{T}_{5}: 2 \mathrm{~L}$ ha-1 (GLU),

$\mathrm{T}_{6}: 4 \mathrm{~L} \mathrm{ha}^{-1}$ (GLU). WL: WeedLock, GLY: glyphosate isopropyl-amine, GLU: glufosinate-ammonium.

Table 8. Sorenson's index of similarity in weed species among different herbicides treatments.

\begin{tabular}{cccccccc}
\hline Treatments & $\mathbf{T}_{\mathbf{0}}$ & $\mathbf{T}_{\mathbf{1}}$ & $\mathbf{T}_{\mathbf{2}}$ & $\mathbf{T}_{\mathbf{3}}$ & $\mathbf{T}_{\mathbf{4}}$ & $\mathbf{T}_{\mathbf{5}}$ & $\mathbf{T}_{\mathbf{6}}$ \\
\hline $\mathrm{T}_{0}$ & - & 59.95 & 59.27 & 53.72 & 60.81 & 67.79 & 45.96 \\
$\mathrm{~T}_{1}$ & 59.95 & - & 56.46 & 46.35 & 57.93 & 56.52 & 38.94 \\
$\mathrm{~T}_{2}$ & 59.27 & 56.46 & - & 61.19 & 59.88 & 66.29 & 49.11 \\
$\mathrm{~T}_{3}$ & 53.72 & 46.35 & 61.19 & - & 50.85 & 53.67 & 46.87 \\
$\mathrm{~T}_{4}$ & 60.81 & 57.93 & 59.88 & 50.85 & - & 59.80 & 61.36 \\
$\mathrm{~T}_{5}$ & 67.79 & 56.52 & 66.29 & 53.67 & 59.80 & - & 57.44 \\
$\mathrm{~T}_{6}$ & 45.96 & 38.94 & 49.11 & 46.87 & 61.36 & 57.44 & - \\
\hline
\end{tabular}

Here, $\mathrm{T}_{0}$ : untreated (control), $\mathrm{T}_{1}: 1345.50 \mathrm{~L} \mathrm{ha}^{-1}(\mathrm{WL}), \mathrm{T}_{2}: 2691.00 \mathrm{~L} \mathrm{ha}^{-1}(\mathrm{WL}), \mathrm{T}_{3}: 2 \mathrm{~L} \mathrm{ha}^{-1}(\mathrm{GLY}), \mathrm{T}_{4}$ : $4 \mathrm{~L} \mathrm{ha}^{-1}(\mathrm{GLY}), \mathrm{T}_{5}: 2 \mathrm{~L} \mathrm{ha}^{-1}$ (GLU), $\mathrm{T}_{6}: 4 \mathrm{~L} \mathrm{ha}^{-1}$ (GLU). WL: WeedLock, GLY: glyphosate isopropyl-amine, GLU: glufosinate-ammonium.

3.2.3. Effects of WeedLock, Glyphosate Isopropyl-Amine, and Glufosinate-Ammonium on Injury Scale of Weed Composition

The efficacy of all herbicides was assessed as the impacts based on the visual injury of total weed composition in the experimental plots in the field. Visual injury assessment was conducted at $1,3,7,14,21,28,35$, and 60 DAS, and it was found that the weed control per- 
centage/injury level was influenced significantly $(p \leq 0.05)$ by the application of WeedLock, glyphosate isopropyl-amine, and glufosinate-ammonium compared to untreated (control) (Table 9). The visual injury scales of herbicide-treated plots were significantly greater than the untreated (control). At 1 DAS, plots treated with WeedLock in $\mathrm{T}_{1}\left(1345.50 \mathrm{~L} \mathrm{ha}^{-1}\right)$ and $\mathrm{T}_{2}$ (2691.00 $\left.\mathrm{L} \mathrm{ha}^{-1}\right)$ exhibited severe damage with an injury scale rating 7.75 and 8.00, respectively, which were significantly different from other treatments. Similarly, at 3 DAS, $\mathrm{T}_{1}$ and $\mathrm{T}_{2}$ completely killed (100\% control) all weed species with an injury rating scale of 9.00 over the other treatments (Figure 1). At 7 DAS, complete weed control was observed in all herbicide treatments. At 14 DAS, a reduction in weed control was starting to occur in WeedLock-treated plots, while glyphosate isopropyl-amine and glufosinate-ammonium remained highly effective. Further reduction in control efficacy was observed at 21 DAS and onwards in all herbicide treatments. However, the systemic herbicide glyphosate isopropyl-amine maintained its high efficacy, having a minimum of $70 \%$ weed control (injury scale of 7.00). On the other hand, both contact herbicides WeedLock and glufosinateammonium, experienced a great reduction in their control efficacy starting from 21 DAS onwards. Meanwhile, most of the weeds started to re-grow or recover from the herbicide injury at 60 DAS in all herbicide-treated plots. As a result, no significant differences were observed between the herbicide treatments and untreated (control).

Table 9. Effects of WeedLock, glyphosate isopropyl-amine, and glufosinate-ammonium on injury scale of mixed weed composition at $1,3,7,14,21,28,35$, and 60 days after spray.

\begin{tabular}{|c|c|c|c|c|c|c|c|c|}
\hline \multirow[t]{2}{*}{ Treatments } & \multicolumn{8}{|c|}{ Visual Injury (Scale) } \\
\hline & 1 DAS & 3 DAS & 7 DAS & 14 DAS & 21 DAS & 28 DAS & 35 DAS & 60 DAS \\
\hline $\mathrm{T}_{0}$ & $1.00 \mathrm{c} \pm 0$ & $1.00 \mathrm{~d} \pm 0$ & $1.00 \mathrm{~b} \pm 0$ & $1.00 \mathrm{~d} \pm 0$ & $1.00 \mathrm{e} \pm 0$ & $1.00 \mathrm{e} \pm 0$ & $1.00 \mathrm{e} \pm 0$ & $1.00 \mathrm{a} \pm 0$ \\
\hline $\mathrm{T}_{1}$ & $7.75 a \pm 0.25$ & $9.00 \mathrm{a} \pm 0$ & $9.00 \mathrm{a} \pm 0$ & $7.75 c \pm 0.25$ & $6.75 \mathrm{~d} \pm 0.25$ & $6.25 \mathrm{~d} \pm 0.25$ & $5.00 \mathrm{~d} \pm 0.41$ & $1.00 \mathrm{a} \pm 0$ \\
\hline $\mathrm{T}_{2}$ & $8.00 \mathrm{a} \pm 0$ & $9.00 \mathrm{a} \pm 0$ & $9.00 \mathrm{a} \pm 0$ & $8.25 b \pm 0.25$ & $7.25 \mathrm{~cd} \pm 0.25$ & $6.50 \mathrm{~cd} \pm 0.29$ & $5.75 \mathrm{~cd} \pm 0.25$ & $1.00 \mathrm{a} \pm 0$ \\
\hline $\mathrm{T}_{3}$ & $2.00 \mathrm{~b} \pm 0$ & $2.00 \mathrm{c} \pm 0$ & $9.00 \mathrm{a} \pm 0$ & $9.00 \mathrm{a} \pm 0$ & $8.25 \mathrm{ab} \pm 0.25$ & $7.50 \mathrm{ab} \pm 0.29$ & $7.00 \mathrm{ab} \pm 0.41$ & $1.00 \mathrm{a} \pm 0$ \\
\hline $\mathrm{T}_{4}$ & $2.00 \mathrm{~b} \pm 0$ & $2.00 \mathrm{c} \pm 0$ & $9.00 \mathrm{a} \pm 0$ & $9.00 \mathrm{a} \pm 0$ & $8.75 a \pm 0.25$ & $7.75 a \pm 0.25$ & $7.25 a \pm 0.25$ & $1.00 \mathrm{a} \pm 0$ \\
\hline $\mathrm{T}_{5}$ & $2.25 b \pm 0.25$ & $3.00 \mathrm{~b} \pm 0$ & $9.00 \mathrm{a} \pm 0$ & $9.00 \mathrm{a} \pm 0$ & $7.75 b c \pm 0.25$ & $6.75 b c d \pm 0.25$ & $6.25 b c \pm 0.25$ & $1.00 \mathrm{a} \pm 0$ \\
\hline $\mathrm{T}_{6}$ & $2.50 \mathrm{~b} \pm 0.29$ & $3.25 b \pm 0.25$ & $9.00 \mathrm{a} \pm 0$ & $9.00 \mathrm{a} \pm 0$ & $8.25 \mathrm{ab} \pm 0.25$ & $7.25 \mathrm{abc} \pm 0.48$ & $6.75 \mathrm{ab} \pm 0.25$ & $1.00 \mathrm{a} \pm 0$ \\
\hline
\end{tabular}

Data are expressed as mean \pm standard error. Means having the same letter among the treatments are not significantly different at $p<0.05$. Here, $\mathrm{T}_{0}$ : untreated (control), $\mathrm{T}_{1}: 1345.50 \mathrm{~L} \mathrm{ha}^{-1}(\mathrm{WL}), \mathrm{T}_{2}: 2691.00 \mathrm{~L} \mathrm{ha}^{-1}(\mathrm{WL}), \mathrm{T}_{3}: 2 \mathrm{~L} \mathrm{ha}^{-1}(\mathrm{GLY}), \mathrm{T}_{4}: 4 \mathrm{~L} \mathrm{ha}^{-1}(\mathrm{GLY}), \mathrm{T}_{5}: 2 \mathrm{~L} \mathrm{ha}{ }^{-1}(\mathrm{GLU})$, $\mathrm{T}_{6}: 4 \mathrm{~L} \mathrm{ha}^{-1}$ (GLU). WL: WeedLock, GLY: glyphosate isopropyl-amine, GLU: glufosinate-ammonium.

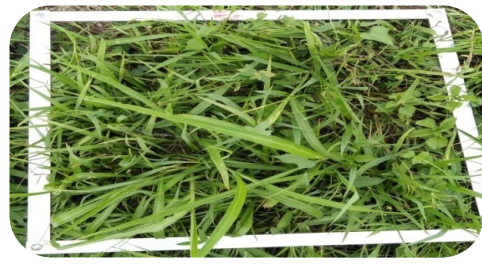

Untreated (control)

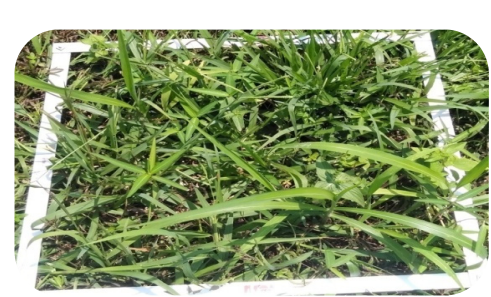

Untreated (control)

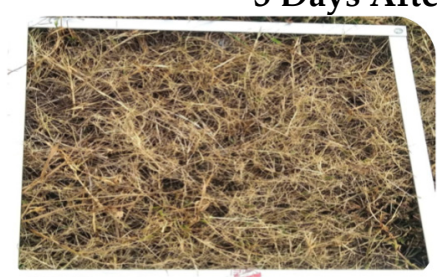

$\mathrm{T}_{1}: 1345.50 \mathrm{~L} \mathrm{ha}^{-1}$

(WeedLock)

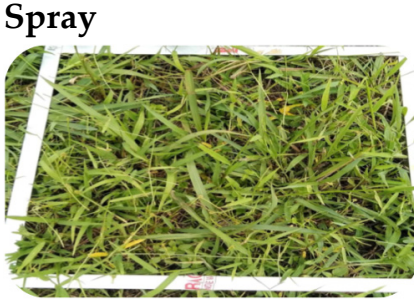

$\mathrm{T}_{3}$ : 2 L ha $^{-1}$ (glyphosate)

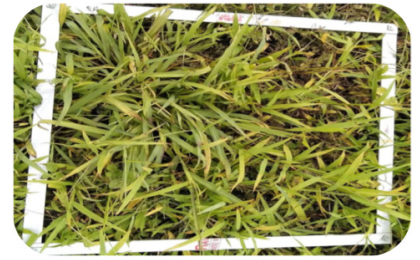

$\mathrm{T}_{5}: 2 \mathrm{~L} \mathrm{ha}^{-1}$ (glufosinate)

7 Days After Spray
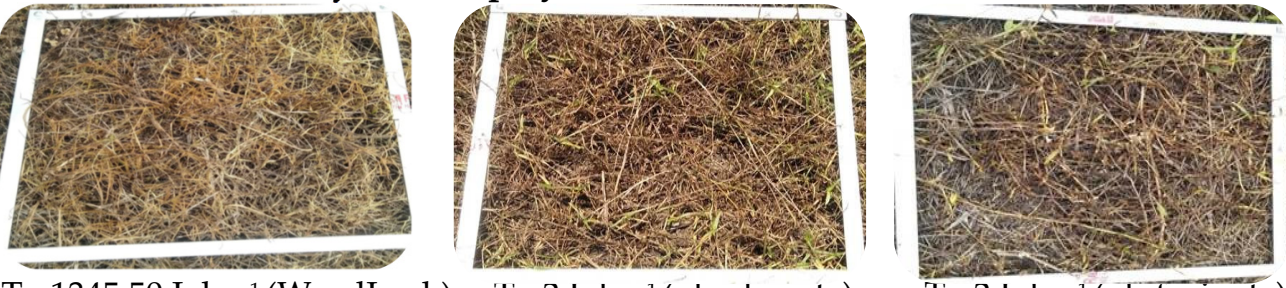

$\mathrm{T}_{5}: 2 \mathrm{~L} \mathrm{ha}^{-1}$ (glutosinate)

Figure 1. Effect WeedLock, glyphosate isopropyl-amine, and glufosinate-ammonium on mixed weed composition at 3 and 7 days after spray. 
3.2.4. Effects of WeedLock, Glyphosate Isopropyl-Amine, and Glufosinate-Ammonium on the Fresh and Dry Weight of Weed Composition

Weed fresh and dry weights were significantly $(p \leq 0.05)$ lower compared to untreated (control) at all the applied doses of WeedLock, glyphosate isopropyl-amine, and glufosinateammonium (Table 10). This level of significance was observed at 35 and 60 days after herbicide application. $\mathrm{T}_{1}$ (1345.50 L ha ${ }^{-1}$ WeedLock) produced the lowest fresh weight reduction of $43.29 \%$ at 35 DAS compared to untreated (control). The highest reduction of fresh weight, $66.18 \%$, occurred in $\mathrm{T}_{4}\left(4 \mathrm{~L} \mathrm{ha}^{-1}\right.$ glyphosate isopropyl-amine) followed by $\mathrm{T}_{6}\left(4 \mathrm{~L} \mathrm{ha}^{-1}\right.$ glufosinate-ammonium) and $\mathrm{T}_{3}\left(2 \mathrm{~L} \mathrm{ha}^{-1}\right.$ glyphosate isopropyl-amine), which caused $65.32 \%$ and $60.28 \%$ reductions, respectively, compared to untreated (control) at 35 DAS. Meanwhile, a higher dose of glyphosate isopropyl-amine $\left(\mathrm{T}_{4}-4 \mathrm{~L} \mathrm{ha}^{-1}\right.$ ) and glufosinate-ammonium $\left(\mathrm{T}_{6}-4 \mathrm{~L} \mathrm{ha}^{-1}\right)$ produced lower dry weight in weeds, namely 27.79 and $28.07 \mathrm{~g}$, respectively, at 35 DAS while $40.38 \mathrm{~g}$ was recorded for WeedLock $\left(\mathrm{T}_{2}-\right.$ $\left.2691.00 \mathrm{~L} \mathrm{ha}^{-1}\right)$. At $60 \mathrm{DAS}$, the dry weight reduction ranged from $23.75 \%$ to $40.03 \%$ compared to untreated (control), with the highest was recorded in $\mathrm{T}_{4}$ ( $4 \mathrm{~L} \mathrm{ha}^{-1}$ glyphosate isopropyl-amine) and the lowest was in $\mathrm{T}_{1}\left(1345.50 \mathrm{~L} \mathrm{ha}^{-1}\right.$ WeedLock).

Table 10. Effects of WeedLock, glyphosate isopropyl-amine, and glufosinate-ammonium on fresh $\left(\mathrm{g} / 0.25 \mathrm{~m}^{2}\right)$ and dry weights $\left(\mathrm{g} / 0.25 \mathrm{~m}^{2}\right)$ of mixed weed composition.

\begin{tabular}{ccccc}
\hline \multirow{2}{*}{ Treatments } & \multicolumn{2}{c}{ 35 DAS } & \multicolumn{2}{c}{ 60 DAS } \\
\cline { 2 - 5 } & Fresh Weight (g) & Dry Weight (g) & Fresh Weight (g) & Dry Weight (g) \\
\hline $\mathrm{T}_{0}$ & $657.98 \mathrm{a} \pm 3.38$ & $82.98 \mathrm{a} \pm 1.67$ & $861.48 \mathrm{a} \pm 3.31$ & $121.22 \mathrm{a} \pm 2.28$ \\
$\mathrm{~T}_{1}$ & $373.09 \mathrm{~b} \pm 2.15$ & $44.16 \mathrm{~b} \pm 1.84$ & $688.23 \mathrm{~b} \pm 2.29$ & $92.41 \mathrm{~b} \pm 1.53$ \\
$\mathrm{~T}_{2}$ & $296.19 \mathrm{c} \pm 2.48$ & $40.38 \mathrm{bc} \pm 1.77$ & $664.38 \mathrm{c} \pm 2.07$ & $90.69 \mathrm{~b} \pm 1.81$ \\
$\mathrm{~T}_{3}$ & $261.31 \mathrm{~d} \pm 3.24$ & $29.16 \mathrm{~d} \pm 1.45$ & $566.32 \mathrm{f} \pm 2.21$ & $76.02 \mathrm{de} \pm 2.41$ \\
$\mathrm{~T}_{4}$ & $222.49 \mathrm{e} \pm 2.88$ & $27.79 \mathrm{~d} \pm 1.24$ & $527.76 \mathrm{~g} \pm 3.25$ & $72.51 \mathrm{e} \pm 2.00$ \\
$\mathrm{~T}_{5}$ & $264.43 \mathrm{~d} \pm 2.98$ & $35.79 \mathrm{c} \pm 1.57$ & $601.01 \mathrm{~d} \pm 3.61$ & $83.35 \mathrm{c} \pm 1.70$ \\
$\mathrm{~T}_{6}$ & $228.12 \mathrm{e} \pm 2.63$ & $28.07 \mathrm{~d} \pm 1.45$ & $584.56 \mathrm{e} \pm 2.94$ & $80.96 \mathrm{~cd} \pm 1.07$ \\
\hline
\end{tabular}

Data are expressed as mean \pm standard error. Means having the same letter among the treatments are not significantly different at $p<0.05$. Here, $\mathrm{T}_{0}$ : untreated (control), $\mathrm{T}_{1}: 1345.50 \mathrm{~L} \mathrm{ha}^{-1}(\mathrm{WL}), \mathrm{T}_{2}: 2691.00 \mathrm{~L} \mathrm{ha}^{-1}(\mathrm{WL})$ $\mathrm{T}_{3}: 2 \mathrm{~L} \mathrm{ha}^{-1}$ (GLY), $\mathrm{T}_{4}: 4 \mathrm{~L} \mathrm{ha}^{-1}(\mathrm{GLY}), \mathrm{T}_{5}: 2 \mathrm{~L} \mathrm{ha}^{-1}$ (GLU), $\mathrm{T}_{6}: 4 \mathrm{~L} \mathrm{ha}^{-1}$ (GLU). WL: WeedLock, GLY: glyphosate isopropyl-amine, GLU: glufosinate-ammonium.

3.2.5. Effects of WeedLock, Glyphosate Isopropyl-Amine, and Glufosinate-Ammonium on Weed Control Efficiency of Weed Composition

Weed control efficiency was significantly $(p \leq 0.05)$ influenced by different application rates of WeedLock, glyphosate isopropyl-amine, and glufosinate-ammonium at 35 and 60 DAS (Figure 2). A significant difference was observed at 35 DAS between $\mathrm{T}_{1}$ (1345.50 L ha ${ }^{-1}$ WeedLock), $\mathrm{T}_{3}$ (2 L ha ${ }^{-1}$ glyphosate isopropyl-amine), and $\mathrm{T}_{5}\left(2 \mathrm{~L} \mathrm{ha}^{-1}\right.$ glufosinate-ammonium), and they provided an excellent weed control efficiency that ranged from $46.82 \%$ to $66.72 \%$ compared to untreated (control). Weed control efficiency was recorded by more than $65 \%$ at the rate of $\mathrm{T}_{4}\left(4 \mathrm{~L} \mathrm{ha}^{-1}\right.$ glyphosate isopropyl-amine $)$ and $\mathrm{T}_{6}$ (4 L ha ${ }^{-1}$ glufosinate-ammonium), whereas $\mathrm{T}_{2}$ (2691.00 $\mathrm{L} \mathrm{ha}^{-1}$ WeedLock) produced more than $50 \%$ efficacy. The application of glyphosate isopropyl-amine and glufosinateammonium at different doses recorded almost similar results of $64.72 \%$ to $66.47 \%$ and $56.91 \%$ to $66.05 \%$, respectively. Lower weed control efficiency was observed at 60 DAS at all applied herbicides. The highest weed control $(40.03 \%)$ occurred in $\mathrm{T}_{4}\left(4 \mathrm{~L} \mathrm{ha}^{-1}\right.$ glyphosate isopropyl-amine) followed by $\mathrm{T}_{3}\left(2 \mathrm{~L} \mathrm{ha}^{-1}\right.$ glyphosate isopropyl-amine $)$ and $\mathrm{T}_{6}$ (4 L ha ${ }^{-1}$ glufosinate-ammonium), which caused $37.15 \%$ and $33.15 \%$ weed control, respectively, compared to untreated (control). 


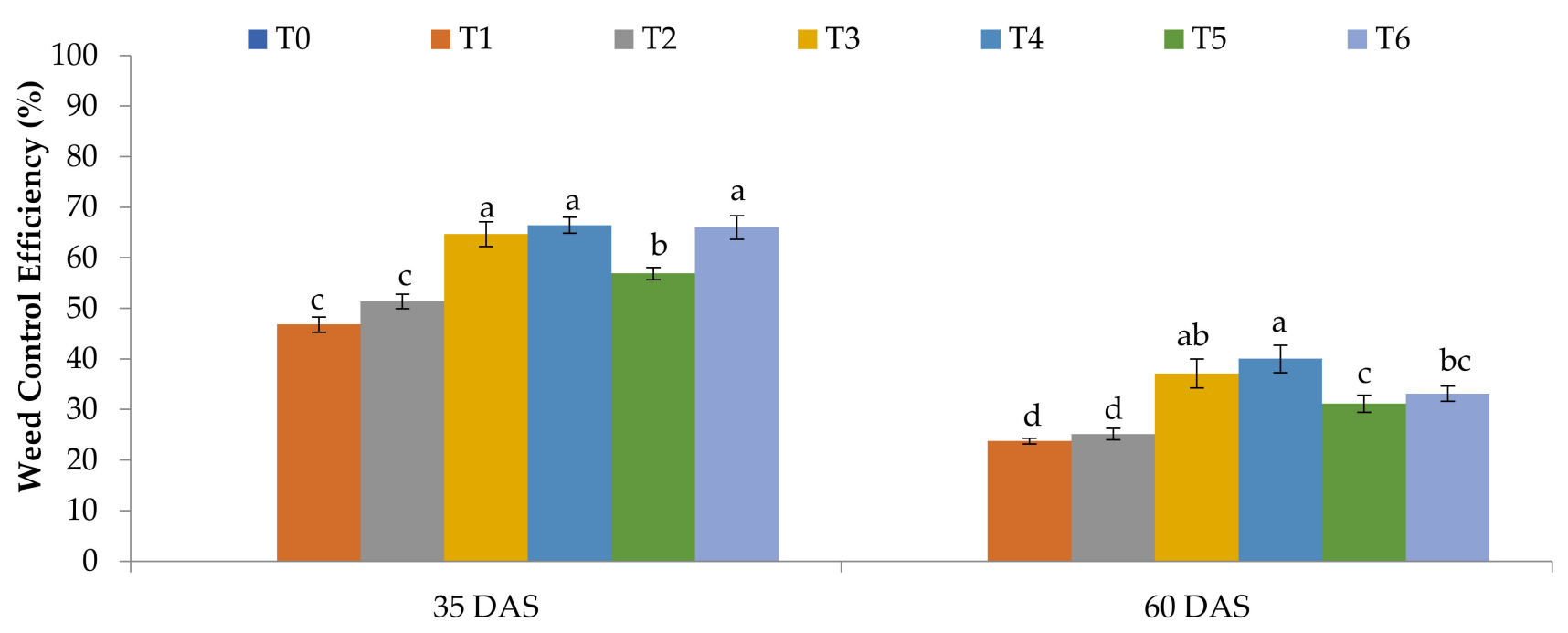

Figure 2. Weed control efficiency after treatment with herbicide at 35 and 65 DAS. Means having the same letter among the treatments are not significantly different at $p<0.05$. Here, $\mathrm{T}_{0}$ : untreated (control), $\mathrm{T}_{1}: 1345.50 \mathrm{~L} \mathrm{ha}^{-1}(\mathrm{WL}), \mathrm{T}_{2}: 2691.00 \mathrm{~L}$ ha ${ }^{-1}$ (WL), $\mathrm{T}_{3}: 2 \mathrm{~L} \mathrm{ha}^{-1}$ (GLY), $\mathrm{T}_{4}: 4 \mathrm{~L} \mathrm{ha}^{-1}$ (GLY), $\mathrm{T}_{5}: 2 \mathrm{~L} \mathrm{ha}^{-1}$ (GLU), $\mathrm{T}_{6}: 4 \mathrm{~L} \mathrm{ha}^{-1}$ (GLU). WL: WeedLock, GLY: glyphosate isopropyl-amine, GLU: glufosinate-ammonium.

\section{Discussion}

Weed management is an essential agronomic practice in agricultural production. Herbicides are the convenient, quick, and arguably the most economical weapon against weeds. In sustainable agriculture, bioherbicides can be used effectively to control economically significant weeds. The study results confirmed that the application of WeedLock, glyphosate isopropyl-amine, and glufosinate-ammonium significantly influenced the weed control and fresh and dry weight reduction of tested weeds. WeedLock bioherbicide showed similar weed control efficacy compared to glyphosate isopropyl-amine and glufosinate-ammonium in the glasshouse for A. conyzoides, E. hirta, and E. indica, but slightly lower efficacy was observed for A. compressus, C. iria, and F. miliacea at the recommended rate $\left(\mathrm{T}_{2}\right)$. The weed control efficiency of WeedLock, glyphosate isopropyl-amine, and glufosinate-ammonium varied among the six weed species, and A. conyzoides was found more sensitive to tested herbicides than the other weeds. Dry weight impacted the weed growth reduction, which mirrored the overall capacity to restrain the weed growth and development in contrast with untreated (control). In the glasshouse experiment, the reduction in dry weight was associated with a decrease in plant growth caused by bioherbicide (WeedLock) and chemical herbicides (glyphosate isopropyl-amine and glufosinate-ammonium). For A. conyzoides and E. indica, WeedLock produced similar weed control efficiency with glyphosate isopropylamine and glufosinate-ammonium. The efficacy of WeedLock increased with increases in the application rate. Similarly, the extract phytotoxicity level of $P$ hysterophorus, C. rutidosperma, and Borreria alata (Aubl.) DC. increased with increasing their concentration and showed a promising inhibitory effect on A. conyzoides and E. hirta [24]. At recommended $\left(\mathrm{T}_{2}\right)$ and higher rates $\left(\mathrm{T}_{3}\right)$, WeedLock produced similar efficacy as glyphosate isopropyl-amine and glufosinate-ammonium.

WeedLock, glyphosate isopropyl-amine, and glufosinate-ammonium possess the nonselective character to the tested crops by causing considerable injury. An injury, such as growth stunting, chlorosis, and burn-down effect, followed by death, was evident with WeedLock, glyphosate isopropyl-amine, and glufosinate-ammonium at 21 days after spray. Moreover, at a lower/half application rate, WeedLock showed low to moderate injury symptoms in the examined crops, while complete killed/dead was observed in glyphosate isopropyl-amine and glufosinate-ammonium. Crop phytotoxicity might depend on application rate, the growth stage of the crop, and other environmental factors that might affect the absorption, translocation, and metabolism of herbicide. All the examined 
crops were completely killed/dead with an injury scale rating 9.00 by all applied doses of glyphosate isopropyl-amine and glufosinate-ammonium over 21 DAS. Glyphosate isopropyl-amine is a systematic non-selective herbicide that can disturb the essential amino acid synthesis [32,33], and glufosinate-ammonium is a non-selective, predominantly contact with restricted systemic action herbicide that can prevent nitrogen metabolism in plant tissue [34,35]. Our study also found the non-selective characteristics of WeedLock, glyphosate isopropyl-amine, and glufosinate-ammonium, as all the examined crops were very heavily injured/dead.

The field trial experiment provided further confirmation for the bioherbicidal potential of WeedLock compared to glyphosate isopropyl-amine and glufosinate-ammonium as observed in the glasshouse experiment. In our field trial study, 32 weed species were identified, comprising broadleaves (13), grasses (13), and sedges (6). The most dominant weed species in the experimental plots were E. ruderalis, C. rutidosperma, A. conyzoides, $C$. dactylon, D. ciliaris, B. mutica, and C. iria. A close similarity in weed species was observed among all the treatments, especially between $\mathrm{T}_{0}$ (untreated), $\mathrm{T}_{2}$ (2691.00 L ha ${ }^{-1}$ WeedLock), $\mathrm{T}_{3}$ (2 L ha ${ }^{-1}$ glyphosate isopropyl-amine), and $\mathrm{T}_{5}\left(2 \mathrm{~L} \mathrm{ha}^{-1}\right.$ glufosinate-ammonium).

As WeedLock is a ready-to-use contact herbicide, it showed great injury at 1, 3, 7, 14, and 21 DAS and enhanced weed control efficiency against mixed weed composition. The highest efficacy of WeedLock with an injury rating scale of 9.00 (complete kill/dead) was observed between 1 and 7 days after spray, and then gradually, the efficacy started to recede. The efficacy of WeedLock bioherbicide was similar to the efficacy produced by glyphosate isopropyl-amine and glufosinate-ammonium at 7 DAS. On the other hand, the detrimental effect of glyphosate isopropyl-amine and glufosinate-ammonium started to occur at 7 to 21 days after spray. Our result is similar to Chang and Liao [36], who mentioned that the lesion symptoms developed slowly among the herbaceous species and died within 7 to 21 days after spraying with glyphosate isopropyl-amine.

WeedLock, glyphosate isopropyl-amine, and glufosinate-ammonium displayed a great reduction in weed control efficiency over 35 and 60 DAS. At 35 DAS, $\mathrm{T}_{2}\left(2691.00 \mathrm{~L} \mathrm{ha}^{-1}\right.$ WeedLock) produced $51.38 \%$, while $\mathrm{T}_{4}\left(4 \mathrm{~L} \mathrm{ha}^{-1}\right.$ glyphosate isopropyl-amine) and $\mathrm{T}_{6}$ (4 L ha ${ }^{-1}$ glufosinate-ammonium) exhibited $66.47 \%$ and $66.05 \%$ weed control efficiency, respectively. Plant-based bioherbicides may involve protein synthesis and decreased protein binding of chlorophyll $\mathrm{a} / \mathrm{b}$ two-fold, affecting photosynthesis by the suppressing of chlorophyll synthesis $[37,38]$. Plant-based bioherbicides reduce the biosynthesis of Oxygen evolving enhancer protein 1, and it influences gas and nutrient exchange in weed species [39]. Bioherbicide efficacy is also strongly influenced by local environmental parameters, such as temperature, moisture, and soil type [40]. Our research also revealed that the efficacy of WeedLock bioherbicide was slightly lower than glyphosate isopropylamine and glufosinate-ammonium at 35 and 60 DAS. In contrast, glyphosate isopropylamine and glufosinate-ammonium are more efficient with high efficacy until 60 DAS. It was previously found that glufosinate-ammonium hindered photosynthesis when in contact with plant foliage and provided good weed control at 7 to 8 DAS, lasting for 30 to 45 days [41-43]. On the other hand, glyphosate isopropyl-amine was absorbed from plant foliage [44], and the affected plants died 7 to 21 days after spray [36]. The symptoms developed slowly but irreversibly $[45,46]$ and showed a higher weed control efficacy, which remained a maximum of 60 to 90 days $[47,48]$.

Our findings indicated that the weed control efficiency of WeedLock was slightly lower compared to glyphosate isopropyl-amine, and glufosinate-ammonium. Ghorbani et al. [49] opined that the effectiveness of bioherbicides depends on the bioactive compound/allelochemical content, plant growth stage, formulation type, spray preparation, application method, type of soil, and environmental factors (light, $\mathrm{CO}_{2}$, temperature, humidity). This might be true in this case, where the WeedLock is a ready-to-use bioherbicide, having a different formulation than glyphosate isopropyl-amine and glufosinateammonium, and as a result could influence weed control efficacy. However, WeedLock bioherbicide requires $1345.50 \mathrm{~L} \mathrm{ha}^{-1}$ to satisfactory acceptable weed control efficacy com- 
parable to that obtained from glyphosate isopropyl-amine and glufosinate-ammonium. At the moment, investigation on enhanced-efficacy of the WeedLock via a reformulation of the current ready-to-use and premix of this promising plant extract bioherbicide is underway.

\section{Conclusions}

The present study indicates that the plant-extract bioherbicide WeedLock has a great phytotoxic effect on the growth and development of tested plants and is also confirmed with herbicidal potential with glyphosate isopropyl-amine and glufosinate-ammonium in both glasshouse and field trials. WeedLock (contact) bioherbicide showed excellent efficacy (100\% weed control) at 1 to 7 days after spraying in comparison to glyphosate isopropyl-amine (systemic) and glufosinate-ammonium (contact, partially systemic). The high efficacy of WeedLock could be characterized as a natural herbicide to control weeds, as well as an alternative to the current synthetic herbicides in both the ornamental and agricultural fields. In addition, studies on the appropriate formulations, residual activity, and mechanism of action are needed for the development of this promising bioherbicide WeedLock.

Author Contributions: Conceptualization, M.S.A.-H. and M.H.; methodology, M.S.A.-H., M.H., and A.M.R.; validation, M.S.A.-H., A.S.M., A.M.R., and M.H.; formal analysis, M.H., M.M., and M.S.A.-H.; investigation, M.S.A.-H., A.S.M., A.M.R., and H.H.; resources, M.S.A.-H., A.M.R., and M.H.; data curation, M.H., M.M., and M.S.A.-H.; writing—original draft preparation, M.H.; writing-review and editing, M.S.A.-H., A.S.M., A.M.R., H.H., M.H., and M.M.; visualization, M.H. and M.S.A.-H.; supervision, M.S.A.-H. and A.S.M.; project administration M.S.A.-H. and A.S.M.; funding acquisition, M.S.A.-H., A.S.M., A.M.R., and H.H. All authors have read and agreed to the published version of the manuscript.

Funding: This research received funding from the Matching Grant: UPM/800-3/3/1/Matching/2018/ 9300451 and EntoGenex Industries Sdn. Bhd. (Matching Grant: Matching/6300931-10201).

Data Availability Statement: Data sharing not applicable.

Acknowledgments: The authors are grateful to Universiti Putra Malaysia for providing the facilities and the scholarship to Mahmudul Hasan (Matching Grant: UPM/800-3/3/1/Matching/2018/9300451) and EntoGenex Industries Sdn. Bhd. for research funding (Matching Grant: Matching/6300931-10201).

Conflicts of Interest: The authors declare no conflict of interest.

\section{References}

1. Juraimi, A.S.; Uddin, M.K.; Anwar, M.P.; Mohamed, M.T.M.; Ismail, M.R.; Man, A. Sustainable weed management in direct seeded rice culture: A review. Aust. J. Crop Sci. 2013, 7, 989-1002.

2. Abouziena, H.F.; Haggag, W.M. Weed control in clean agriculture: A review. Planta Daninha 2016, 34, 377-392. [CrossRef]

3. Heap, I. Herbicide resistant weeds. In Integrated Pest Management; Springer: Dordrecht, The Netherlands, $2014 ;$ pp. $281-301$.

4. Ruzmi, R.; Ahmad-Hamdani, M.S.; Bakar, B.B. Prevalence of herbicide-resistant weed species in Malaysian rice fields: A review. Weed Biol. Manag. 2017, 17, 3-16. [CrossRef]

5. Hussain, M.I.; Reigosa, M.J. Higher peroxidase activity, leaf nutrient contents and carbon isotope composition changes in Arabidopsis thaliana are related to rutin stress. J. Plant Physiol. 2014, 171, 1325-1333. [CrossRef]

6. Islam, A.K.M.M.; Yeasmin, S.; Qasem, J.R.S.; Juraimi, A.S.; Anwar, M.P. Allelopathy of Medicinal Plants: Current Status and Future Prospects in Weed Management. Agric. Sci. 2018, 9, 1569-1588. [CrossRef]

7. Motmainna, M.; Juraimi, A.S.; Uddin, M.K.; Asib, N.B.; Islam, A.K.M.M.; Hasan, M. Assessment of allelopathic compounds to develop new natural herbicides: A review. Allelopath. J. 2021, 52, 19-37. [CrossRef]

8. El-Darier, S.M.; Abdelaziz, H.A.; ZeinEl-Dien, M.H. Effect of soil type on the allelotoxic activity of Medicago sativa L. residues in Vicia faba L. agroecosystems. J. Taibah Univ. Sci. 2014, 8, 84-89. [CrossRef]

9. Hosni, K.; Hassen, I.; Sebei, H.; Casabianca, H. Secondary metabolites from Chrysanthemum coronarium (Garland) flowerheads: Chemical composition and biological activities. Ind. Crop Prod. 2013, 44, 263-271. [CrossRef]

10. Kremer, R.J. The role of bioherbicides in weed management. Biopestic. Int. 2005, 1, 127-141.

11. Bailey, K.L. The bioherbicide approach to weed control using plant pathogens. In Integrated Pest Management; Academic Press: Cambridge, CA, USA, 2014; pp. 245-266.

12. Travlos, I.; Rapti, E.; Gazoulis, I.; Kanatas, P.; Tataridas, A.; Kakabouki, I.; Papastylianou, P. The herbicidal potential of different pelargonic acid products and essential oils against several important weed species. Agronomy 2020, 10, 1687. [CrossRef] 
13. Muñoz, M.; Torres-Pagán, N.; Peiró, R.; Guijarro, R.; Sánchez-Moreiras, A.M.; Verdeguer, M. Phytotoxic effects of three natural compounds: Pelargonic acid, carvacrol, and cinnamic aldehyde, against problematic weeds in Mediterranean crops. Agronomy 2020, 10, 791. [CrossRef]

14. Verdeguer, M.; Sánchez-Moreiras, A.M.; Araniti, F. Phytotoxic effects and mechanism of action of essential oils and terpenoids. Plants 2020, 9, 1571. [CrossRef]

15. Hasan, M.; Ahmad-Hamdani, M.S.; Rosli, A.M.; Hamdan, H. Bioherbicides: An Eco-Friendly Tool for Sustainable Weed Management. Plants 2021, 10, 1212. [CrossRef] [PubMed]

16. Travaini, M.L.; Sosa, G.M.; Ceccarelli, E.A.; Walter, H.; Cantrell, C.L.; Carrillo, N.J.; Dayan, F.E.; Meepagala, K.M.; Duke, S.O. Khellin and Visnagin, Furanochromones from Ammi visnaga (L.) Lam., as Potential Bioherbicides. J. Agric. Food Chem. 2016, 64, 9475-9487. [CrossRef]

17. Motmainna, M.; Juraimi, A.S.; Uddin, M.K.; Asib, N.B.; Islam, A.K.M.M.; Hasan, M. Allelopathic potential of Malaysian invasive weed species on Weedy rice (Oryza sativa f. spontanea Roshev). Allelopath. J. 2021, 53, 53-68. [CrossRef]

18. Raveau, R.; Fontaine, J.; Lounès-Hadj Sahraoui, A. Essential oils as potential alternative biocontrol products against plant pathogens and weeds: A review. Foods 2020, 9, 365. [CrossRef] [PubMed]

19. Motmainna, M.; Juraimi, A.S.; Uddin, M.K.; Asib, N.B.; Islam, A.K.M.M.; Ahmad-Hamdani, M.S.; Berahim, Z.; Hasan, M. Physiological and Biochemical Responses of Ageratum conyzoides, Oryza sativa f. spontanea (Weedy Rice) and Cyperus iria to Parthenium hysterophorus Methanol Extract. Plants 2021, 10, 1205. [CrossRef]

20. Jursík, M.; Soukup, J.; Holec, J.; Andr, J. Important aspects of chemical weed control: Environmental factors affecting herbicide efficacy. Listy Cukrov. Rep. 2011, 127, 348.

21. Van Alfen, N.K. Encyclopedia of Agriculture and Food Systems; Academic Press: Cambridge, CA, USA, 2014.

22. Ozkan, T.G.I.; Urusak, E.A.; Appiah, K.S.; Fujii, Y.; Ozkan, I. First Broad Screening of Allelopathic Potential of Wild and Cultivated Plants in Turkey. Plants 2019, 8, 532. [CrossRef] [PubMed]

23. Islam, A.K.M.M.; Hasan, M.M.; Yeasmin, S.; Abedin, M.A.; Kader, M.A.; Rashid, M.H.; Anwar, M.P. Bioassay screening of tropical tree sawdust for allelopathic properties and their field performance against paddy weeds. Fundam. Appl. Agric. 2019, 4, 906-915.

24. Motmainna, M.; Juraimi, A.S.; Uddin, M.K.; Asib, N.B.; Islam, A.K.M.M.; Hasan, M. Bioherbicidal Properties of Parthenium hysterophorus, Cleome rutidosperma and Borreria alata Extracts on Selected Crop and Weed Species. Agronomy 2021, 11, 643. [CrossRef]

25. Burrill, L.C.; Cárdenas, J.; Locatelli, E. Field Manual for Weed Control Research; International Plant Protection Center, Oregon State University: Corvallis, OR, USA, 1976; p. 63.

26. Abdullah, M.R.; Zakaria, N.; Ahmad-Hamdani, M.S.; Juraimi, A.S. Evaluation of Herbicide Efficacy on Weed Control and Grain Yield in Rice Field under Flooded Condition. Plant Arch. 2020, 20, 8163-8169.

27. Barnes, D.E.; Chan, L.G. Common Weeds of Malaysia and Their Control; Ancom Berhad: Petaling Jaya, Malaysia, 1990.

28. Fee, C.G.; Tui, L.C.; Bin, C.S.; Hoy, C.K. Pictorial Guide to Common Weeds of Plantations and Their Control (No. L-0987); Agricultural Crop Trust, ACT: Bonn, Germany, 2013.

29. Juraimi, A.S.; Muhammad Saiful, A.H.; Uddin, M.K.; Anuar, A.R.; Azmi, M. Diversity of weed communities under different water regimes in bertam irrigated direct seeded rice field. Aust. J. Crop Sci. 2011, 5, 595-604.

30. Janiya, J.D.; Moody, K. Weed populations in transplanted and wet-seeded rice as affected by weed control method. Int. J. Pest Manag. 1989, 35, 8-11. [CrossRef]

31. Goldsmith, F.B.; Harisson, C.M.; Morton, A.J. Description and analysis of vegetation. In Methods in Plant Ecology; Moore, P.D., Chapman, S.B., Eds.; Blackwell Scientific Publication: London, UK, 1986; pp. 437-521.

32. Garba, J.; Othman, R.; Hamdani, M.S.A. Adsorption-desorption and leaching potential of glyphosate and aminomethylphosphonic acid in acidic Malaysian soil amended with cow dung and rice husk ash. Environ. Monit. Assess. 2018, 190, 1-15. [CrossRef]

33. Sherwani, S.I.; Arif, I.A.; Khan, H.A. Modes of action of different classes of herbicides. In Herbicides: Physiology of Action, and Safety; IntechOpen: London, UK, 2015; pp. 165-186. [CrossRef]

34. Takano, H.K.; Dayan, F.E. Glufosinate-ammonium: A review of the current state of knowledge. Pest Manag. Sci. 2020, 76, 3911-3925. [CrossRef]

35. Reddy, K.N.; Zablotowicz, R.M.; Bellaloui, N.; Ding, W. Glufosinate effects on nitrogen nutrition, growth, yield, and seed composition in glufosinate-resistant and glufosinate-sensitive soybean. Int. J. Agron. 2011, 1-9. [CrossRef]

36. Chang, S.Y.; Liao, C.H. Analysis of glyphosate, glufosinate and aminomethylphosphonic acid by capillary electrophoresis with indirect fluorescence detection. J. Chromatogr. A 2002, 959, 309-315. [CrossRef]

37. Heide, H.; Kalisz, H.M.; Follmann, H. The oxygen evolving enhancer protein 1 (OEE) of photosystem II in green algae exhibits thioredoxin activity. J. Plant Physiol. 2004, 161, 139-149. [CrossRef]

38. Motmainna, M.; Juraimi, A.S.; Uddin, M.K.; Asib, N.B.; Islam, A.K.M.M.; Ahmad-Hamdani, M.S.; Hasan, M. Phytochemical Constituents and Allelopathic Potential of Parthenium hysterophorus L. in Comparison to Commercial Herbicides to Control Weeds. Plants 2021, 10, 1445. [CrossRef]

39. Lee, S.M.; Radhakrishnan, R.; Kang, S.M.; Kim, J.H.; Lee, I.Y.; Moon, B.Y.; Yoon, B.W.; Lee, I.J. Phytotoxic mechanisms of bur cucumber seed extracts on lettuce with special reference to analysis of chloroplast proteins, phytohormones, and nutritional elements. Ecotoxicol. Environ. Saf. 2015, 122, 230-237. [CrossRef]

40. Cai, X.; Gu, M. Bioherbicides in organic horticulture. Horticulturae 2016, 2, 3. [CrossRef] 
41. Wibawa, W.; Mohamad, R.B.; Omar, D.; Zain, N.M.; Puteh, A.B.; Awang, Y. Comparative impact of a single application of selected broad spectrum herbicides on ecological components of oil palm plantation. Afr. J. Agric. Res. 2010, 5, 2097-2102. [CrossRef]

42. Hoerlein, G. Glufosinate (phosphinothricin), a natural amino acid with unexpected herbicidal properties. Rev. Environ. Contam. Toxicol. 1994, 138, 73-145. [CrossRef]

43. Riley, P.; Warhurst, M.; Diamand, E.; Barron, H. Health and Environmental Impacts of Glufosinate-Ammonium; PAN UK: Brighton, UK, 2001.

44. Garba, J.; Abd Samsuri, W.; Othman, R.; Hamdani, M.S.A. Evaluation of Adsorptive Characteristics of Cow Dung and Rice Husk Ash for Removal of Aqueous Glyphosate and Aminomethylphoshonic Acid. Sci. Rep. 2019, 9, 1-10. [CrossRef] [PubMed]

45. Collins, S.C. Chemical control of grassy weeds. In Tropical Grassy Weeds; Baker, F.W.G., Terry, P.J., Eds.; CAB: Wallingford, UK, 1991.

46. Hoss, N.E.; Al-Khatib, K.; Peterson, D.E.; Loughin, T.M. Efficacy of glyphosate, glufosinate, and imazethapyr on selected weed species. Weed Sci. 2003, 51, 110-117. [CrossRef]

47. Mohamad, R.; Mohayidin, M.G.; Wibaya, W.; Juraimi, A.S.; Lassim, M.M. Management of mixed weeds in young oil-palm plantation with selected broad-spectrum herbicides. Pertanika J. Trop. Agric. Sci. 2010, 33, 193-203.

48. Wibawa, W.; Mohayidin, M.G.; Mohamad, R.B.; Juraimi, A.S.; Omar, D. Efficacy and cost-effectiveness of three broad-spectrum herbicides to control weeds in immature oil palm plantation. Pertanika J. Trop. Agric. Sci. 2010, 33, $233-241$.

49. Ghorbani, R.; Seel, W.; Rashed, M.H.; Leifert, C. Effect of plant age, temperature and humidity on virulence of Ascochyta caulina on common lambsquarters (Chenopodium album). Weed Sci. 2006, 54, 526-531. [CrossRef] 